\title{
El Corredor Magmático Intracratónico Pérmico-Triásico de la provincia de La Pampa, Argentina: nuevas edades U-Pb SHRIMP, composición isotópica de Hf e implicancias geodinámicas
}

\section{Carlos J. Chernicoff ${ }^{1, *}$, Eduardo O. Zappettini' ${ }^{2}$, João O. Santos ${ }^{3}$ y Neal McNaughton ${ }^{4}$}

\author{
${ }^{1}$ Consejo Nacional de Investigaciones Científicas y Técnicas, Servicio Geológico-Minero Argentino, \\ Av. Gral Paz 5445, San Martín, provincia de Buenos Aires, Argentina. \\ ${ }^{2}$ Servicio Geológico-Minero Argentino, Av. Gral Paz 5445, San Martín, provincia de Buenos Aires, Argentina. \\ ${ }^{3}$ Center of Exploration Targeting, University of Western Australia, 35 Stirling Highway, Perth, WA, Australia. \\ ${ }^{4}$ John de Laeter Centre, Curtin University, Kent Street, Bentley 9102, Australia. \\ ${ }^{*}$ cc_jorge@yahoo.com.ar
}

\section{RESUMEN}

Se ha realizado un estudio y revisión de las unidades magmáticas que integran el Corredor Magmático Intracratónico PérmicoTriásico de La Pampa (CMPT-LP), centro-sur de Argentina. Se dan a conocer nuevas edades $\mathrm{U}-\mathrm{Pb}$ SHRIMP, composición isotópica de Hf y composición química de algunas unidades integrantes del CMPT-LP: 1) Sienogranito Chacharramendi, edad $254.7 \pm 1.3 \mathrm{Ma}$ (Pérmico tardío, Lopingiano), edad modelo Hf de 1640 Ma con $\varepsilon_{\mathrm{Hf}}$ promedio de -5.68 ; 2) Riolita Lihue Calel, $239.3 \pm 1.5 \mathrm{Ma}$ (Triásico Medio), edad modelo Hf de $1640 \mathrm{Ma}$ con $\varepsilon_{\mathrm{Hf}}$ promedio de -5.56. Esto indica que las dos unidades datadas fueron cristalizadas a partir de un fundido mantélico que incorporó corteza de edad statheriana tardía hasta calymmiana, tomando en cuenta también la edad modelo Nd de $1483 \mathrm{Ma}$ del sector más distal (sudoriental) (Granito López Lecube) del CMPTLP obtenida por otros autores.

Nuevos datos geoquímicos aquí aportados, analizados junto con datos geoquímicos previos, denotan la impronta o huella anorogénica -ambiente de intraplaca- del corredor magmático CMPT-LP, destacándose su consistencia con los granitos del tipo $\mathrm{A}_{1}$ debido a la similitud de sus fuentes con aquéllas de las que derivan los basaltos de islas oceánicas (OIB), como queda evidenciado, p. ej., en los cocientes $\mathrm{Y} / \mathrm{Nb}<1.2$. La pertenencia de las rocas del CMPT-LP mayoritariamente al tipo de alto potasio también es consistente con su ocurrencia en un ambiente tectónico de intraplaca. El ambiente anorogénico del CMPT-LP contrasta con aquél de la etapa tardía del Grupo Choiyoi propiamente dicho, que si bien también está asociada a extensión pérmica tardía - triásica temprana, ocurre en el contexto orogénico propio del Arco Magmático Choiyoi. Por tal razón se analiza la conveniencia de excluir el CMPT-LP del Grupo Choiyoi, en el que hasta ahora estaba incluido. Asimismo se comparan brevemente los marcos tectónicos de las fajas orogénicas lindantes del CMPT-LP: fajas de San Rafael (o Sanrafaélica, o Arco-Retroarco Choiyoi) y Gondwánides Norpatagónicos.

Palabras clave: corredor magmático intracratónico; edad U-Pb SHRIMP; isótopos Hf; Pérmico-Triásico; provincia La Pampa, Argentina.

\section{ABSTRACT}

A study and review of the magmatic units that make up the PermianTriassic Intracratonic Magmatic Corridor of La Pampa (CMPT-LP), south-central Argentina, has been carried out. New U-Pb SHRIMP ages, $H$ isotopic composition, and chemical composition of some units of the CMPT-LP have been obtained, i.e. 1) Chacharramendi Syenogranite, age $254.7 \pm 1.3 \mathrm{Ma}$ (Late Permian, Lopingian), Hf model age of $1640 \mathrm{Ma}$ with $\varepsilon_{H f}$ average of $\left.-5.68 ; 2\right)$ Lihue Calel Rhyolite, 239.3 $\pm 1.5 \mathrm{Ma}$ (Middle Triassic), Hf model age of $1640 \mathrm{Ma}$ with $\varepsilon_{\mathrm{Hf}}$ average -5.56 . This indicates that the two dated units were crystallized from a mantle-derived melt that incorporated crust of late Statherian up to Calymmian age, taking also into account a Nd model age of $1483 \mathrm{Ma}$ for the more distal (southeasternmost) sector (López Lecube Granite) of the CMPT-LP obtained by other authors.

New geochemical data reported here, analyzed together with previous geochemical data, denote the anorogenic signature -intraplate environment- of the CMPT-LP magmatic corridor, highlighting its consistency with $A_{1}$-type granites due to the similarity of their sources with those from which the oceanic island basalts (OIB) derive, as evidenced, e.g., by $\mathrm{Y} / \mathrm{Nb}$ ratios $<1.2$. The classification of the rocks of the CMPT-LP mainly within the high potassium type is also consistent with its occurrence in 
an intraplate tectonic environment. The magmatism in the CMPT-LP has been considered as part of the Choiyoi Group, which is also related to late Permian to Early Triassic extension. Nevertheless, there is a marked contrast between the anorogenic environment of the CMPT-LP and the orogenic context of the Choiyoi Magmatic Arc. For this reason, the convenience of excluding the CMPT-LP from the Choiyoi Group is analyzed. In addition, the tectonic frames of the orogenic belts adjoining the CMPT-LP-San Rafael (or Sanrafaelic, or Choiyoi Arc-retroarc) and Northpatagonian Gondwanide belts-are compared briefly.

Key words: intracratonic magmatic corridor; U-Pb SHRIMP age; Hf isotopes; Permian-Triassic; La Pampa province, Argentina.

\section{INTRODUCCIÓN}

El corredor magmático pérmico-triásico de la provincia de La Pampa (CMPT-LP) ha sido interpretado como originado tanto en un ambiente post-orogénico como en un ambiente intracratónico (Llambías et al., 2003; Llambías y Sato, 1990; Sato et al., 2015). Con el objeto de esclarecer esta cuestión -y el de comparar el contexto tectónico del CMPT-LP con el de su región circundante- en este trabajo se dan a conocer nuevas edades U-Pb SHRIMP, datos de la composición isotópica de $\mathrm{Hf}$ y de la composición química de unidades que forman parte de este corredor magmático. El CMPT-LP está localizado en la provincia de La Pampa, centro-sur de Argentina, y tiene continuidad en el sur de las provincias de Mendoza y Buenos Aires (Figura 1).

En algunos trabajos anteriores el corredor magmático CMPT-LP fue considerado como post-orogénico (p. ej., Llambías et al., 2003) respecto de la Fase Orogénica San Rafael (FOSR; Llambías et al., 1993) ocurrida en el Pérmico Inferior del margen paleopacífico central de América del Sur. En tanto, en otros trabajos (e.g., Llambías y Sato, 1990; Sato et al., 2015), este corredor magmático ha sido señalado como anorogénico, criterio que se prefiere en el presente artículo debido a la ausencia de deformación compresiva (FOSR ausente) en la roca sedimentaria encajonante pérmica inferior del mismo (Formación Carapacha; p. ej., Tomezzoli y Melchor, 2002; Chernicoff et al., 2008), entre otras consideraciones. Esta ausencia de deformación plegante en la Formación Carapacha contrasta con la deformación plegante causada por la FOSR en el sustrato sedimentario del Grupo Choiyoi propiamente dicho (o Arco Magmático Choiyoi), más hacia el noroeste, en el sector principal de la cuenca de San Rafael (Formación El Imperial, Pensilvánico-Pérmico muy temprano).

Tomando en cuenta el contexto tectónico y ubicación geográfica del corredor magmático CMPT-LP, se analiza la conveniencia de excluirlo del Grupo Choiyoi -de edad contemporánea pero no asociado espacialmente a una región intracratónica- en el que hasta ahora estaba incluido (p. ej., Sato et al., 2015). Si bien el Grupo o Provincia Magmática Choiyoi ha sido muy estudiado desde diversos puntos de vista (petrológico, geoquímico, estructural, tectónico, paleomagnético), de lo que dan cuenta numerosos trabajos recientes (Kleiman y Japas, 2009, Domeier et al., 2011, Tomezzoli et al., 2011, Sato et al., 2015, Poma et al., 2014, Rocher et al., 2015, entre otros), la región del CMPT-LP no ha sido mayormente el objeto principal de estos estudios.

Asimismo se comparan brevemente los marcos tectónicos de las fajas orogénicas lindantes del CMPT-LP -fajas de San Rafael (ó Sanrafaélica, o Arco-Retroarco Choiyoi) y Gondwánides Norpatagónicos-, discutiéndose: 1) la pertinencia de separarlas o deslindarlas temporal y espacialmente entre sí, en contraposición al concepto mayormente aceptado de una evolución transicional entre dichas fajas (Kleiman y Japas, 2009, Pángaro et al., 2016, entre otros), y 2) la connotación que tiene la diferencia evolutiva entre estas dos fajas orogénicas -i.e., carácter acrecional de la Faja Sanrafaélica versus carácter colisional de los Gondwánides Nordpatagónicos (contextos de subducción continua versus terminación del ciclo de Wilson; terminología de Cawood y Buchan, 2007), inclusive con distintos sentidos de la polaridad de la paleo-subducción asociada en cada caso (p. ej., Sato et al., 2015)- para su exploración geológico-minera.

El objetivo inicial de este trabajo es esclarecer el ambiente tectónico en que tuvo lugar el magmatismo pérmico-triásico de la provincia de La Pampa, Argentina, precisar su edad, composición isotópica y distribución geográfica, así como analizar su desvinculación de dos fajas magmáticas Gondwánicas lindantes y parcialmente contemporáneas. En segundo lugar, analizar el diferente marco tectónico de emplazamiento entre estas dos últimas fajas, en contraposición al concepto mayormente aceptado de una evolución transicional entre ellas.

\section{MAGMATISMO GONDWÁNICO}

Una importante faja magmática pérmico-triásica ocurre de manera discontinua a lo largo del margen occidental de Gondwana en América del Sur, con afloramientos desde el Perú central hasta latitudes próximas a los $39^{\circ} \mathrm{S}$ en la Argentina. De oeste a este esta faja se extiende aproximadamente por $600 \mathrm{~km}$ desde el margen Gondwánico hacia el antepaís, alcanzando la longitud de las actuales Sierras Pampeanas y el margen occidental del cratón del Río de la Plata.

Esta faja comprende rocas plutónicas epizonales y volcánicas -incluyendo una voluminosa facies piroclástica-, conjuntamente referidas como provincia magmática Choiyoi en la Argentina y Chile (Kay et al., 1989; Llambías et al., 1993; Llambías, 1999). Esta provincia magmática se habría desarrollado en un contexto que evolucionó desde un arco magmático relacionado a subducción carbonífera superior a pérmica, seguido de un régimen colisional (p. ej., Llambías et al., 2003; Kleiman y Japas, 2009; Rocha-Campos et al., 2011; Charrier et al., 2014; Poma et al., 2014; Rocher et al., 2015; Sato et al., 2015; Pángaro et al., 2016; y trabajos allí citados) sucedido, en el Trasico Inferior, por un magmatismo ácido post-orogénico (p. ej., Llambías, 1999). Desde el Triásico Medio (i.e., post-Choiyoi) la última etapa habría evolucionado transicionalmente a un magmatismo de tipo synrift (Kleiman y Japas, 2009; Rocha-Campos et al., 2011; Poma et al., 2014, entre otros).

En la Argentina, la provincia magmática Choiyoi está expuesta en: 1) la Cordillera Frontal de Mendoza y San Juan, donde constituye los afloramientos más voluminosos de rocas félsicas, y cantidades subordinadas de rocas básicas y mesosilícicas (p. ej., Mpodozis and Kay, 1992; Llambías et al., 1993, Breitkreuz and Zeil, 1994; Rocher et al., 2015) y donde las secuencias volcánicas predominan en la Argentina y las rocas plutónicas en Chile (i.e., Charrier et al., 2014, y referencias allí citadas), aunque algunos plutones importantes tal como el batolito de Colangüil también ocurren en la Argentina (Llambías and Sato, 1990, 1995); 2) la región de la Puna de la provincia de Salta, como afloramientos aislados (p. ej., Zappettini y Blasco, 1998; Page y Zappettini, 1999; Poma et al., 2014) que continúan hacia el noroeste en Chile (p. ej., Munizaga et al., 2008); 3) el basamento de la cuenca Neuquina y en la Cordillera Principal de la región austral de la provincia de Mendoza (p. ej., Llambías et al., 2007; Casé et al., 2008; Barrionuevo et al., 2013); 4) el bloque San Rafael y su continuación austral en la provincia de La Pampa (p. ej., Sruoga y Llambías, 1992; Llambías et al., 2003; Kleiman y Japas, 2009; Rocha-Campos et al., 2011; Chernicoff et al., 2016a), región esta última en donde enfoca su atención el presente trabajo. A su vez, el magmatismo Gondwánico se extiende hacia el sudeste (p. ej., Gregori et al., 2003), al norte patagónico (p. ej., Caminos et al., 1988; Rapela y Llambías, 1985; Pankhurst et al., 1992). 


\section{LAS ROCAS ANALIZADAS}

\section{Sienogranito Chacharramendi (MG88)}

Con el objeto de la realización de análisis isotópicos y geoquímicos se tomaron muestras del Sienogranito Chacharramendi en la proximidad al poblado homónimo de la provincia de La Pampa (-37.32143 S / $-65.71778 \mathrm{O}$ ), donde este cuerpo plutónico aflora muy someramente, y hay una cantera inactiva. Con anterioridad al presente estudio, esta unidad contaba con dos edades triásicas superiores obtenidas por Linares et al. (1980): K-Ar $207 \pm 10 \mathrm{Ma}$, y Rb-Sr $213 \pm 10 \mathrm{Ma}$.

Se trata de un leucosienogranito rosado, fresco, de textura granosa hipidiomórfica, grano mediano y con algunos cristales mayores de hasta $10 \mathrm{~mm}$ de feldespato potásico. Está constituido por feldespato potásico, plagioclasa (oligoclasa media), cuarzo y escasa biotita. Las relaciones geológicas de campo de este cuerpo resultan inciertas debido a que la cobertura sedimentaria moderna impide ver sus límites y contactos.

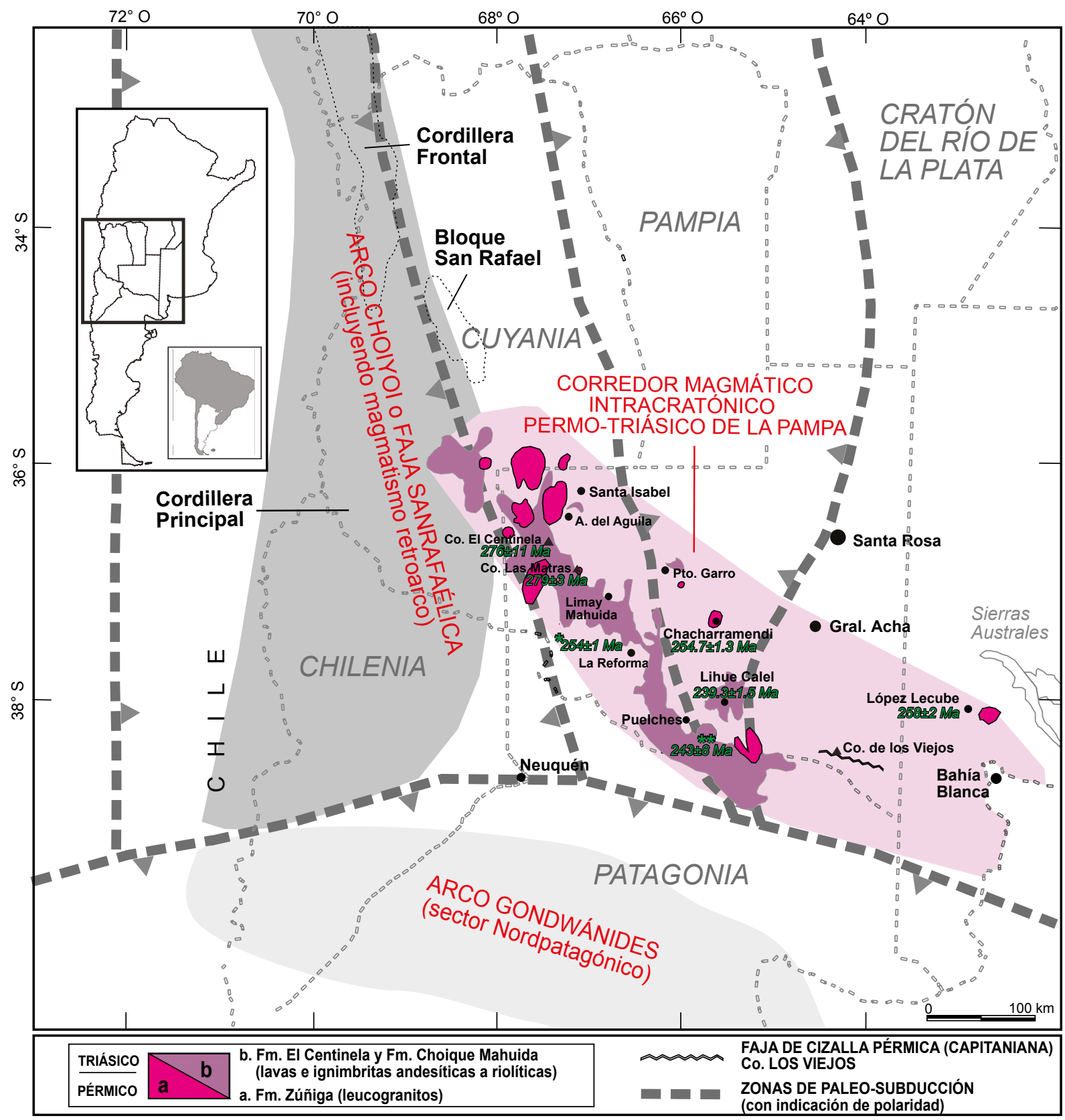

Figura 1. Contexto tectónico del Corredor Magmático Intracratónico Pérmico-Triásico de La Pampa (CMPT-LP). Geología pérmica-triásica simplificada, modificada de Kleiman y Japas (2009), Rocher et al. (2015) y Sato et al. (2015, y referencias allí citadas), entre otros. Límites entre terrenos tectono-estratigráficos (indicados como zonas de paleo-subducción) tomados de Chernicoff y Zappettini (2004). Ubicación de la faja de cizalla pérmica (capitaniana) del Cerro de Los Viejos tomada de Tickyj et al. (1997). Nótese cierta superposición del corredor CMPT-LP con el retroarco Choiyoi (sensu Rocher et al., 2015). Edades: Granito López Lecube (U-Pb SHRIMP en circones, Pankhurst et al., 2006); Traquiandesita El Centinela (U-Pb convencional, dos cristales de circón, Tickyj et al., 2010); Trondhjemita Las Matras (U-Pb SHRIMP en circones, un cristal de circón, Chernicoff et al., 2016b); ${ }^{*}$ un asterisco) sanidina pérmica en toba andesítica eocena de la Formación Gran Salitral (Ar-Ar en sanidina, Melchor, 2002); ** (dos asteriscos) Sienita Estancia El Trabajo (K-Ar en anfíbol, Lagorio et al., 2008); Sienogranito Chacharramendi y Riolita Lihue Calel (U-Pb SHRIMP en circones, este trabajo). 


\section{Riolita Lihue Calel (GA119)}

Asimismo, se tomó una muestra de la Riolita Lihue Calel en la sierra homónima de la provincia de La Pampa (-38.02222 S / -65.58916 O) con el objeto de obtener una confirmación de su edad por el método $\mathrm{U}-\mathrm{Pb}$ SHRIMP en circones, y de establecerse la composición isotópica de Hf de los circones datados. Linares et al. (1980) dataron rocas en esta localidad, obteniendo una edad $238 \pm 5 \mathrm{Ma}$ ( $\mathrm{Rb}$-Sr isócrona en roca total), y una edad $235 \pm 5 \mathrm{Ma}(\mathrm{K}-\mathrm{Ar})$, en tanto que Rapela et al. (1996) establecieron una edad de $240 \pm 2 \mathrm{Ma}(\mathrm{Rb}-\mathrm{Sr}$ isócrona en roca total).

La Riolita Lihue Calel consiste en una espesa sucesión de ca. 1800 $m$ de ignimbritas con abundante cantidad de fenocristales, lavas y brechas en menor proporción, que cuenta con estudios petrológicos y geoquímicos (p. ej., Sruoga y Llambías 1992, Llambías 1973).

\section{DATACIÓN U-Pb SHRIMP}

\section{Metodología}

Las rocas (muestras MG88 y GA119) fueron trituradas, pulverizadas, tamizadas a malla 60, y lavadas para remover las fracciones arcilla y limo. El material restante, correspondiente a arena fina y muy fina, fue secado y procesado por dos líquidos pesados: LST (politungstato de litio, densidad 2.82) y TBE (tetrabromoetano, densidad 2.98). Los concentrados de minerales pesados fueron separados en cuatro fracciones usando un separador magnético Frant $z^{\oplus}$. Usando una inclinación lateral de $5^{\circ}$ y una corriente de 1 Ampere, los granos de circón fueron recogidos de la fracción menos magnética (y titanita de la fracción más magnética). Los granos luego fueron montados en discos de epoxy de $2,5 \mathrm{~cm}$ de diámetro junto con los estándares analíticos. Los montajes se pulieron y recubrieron con carbono para formación de imágenes usando un microscopio electrónico de barrido TESCANVEGA3 en el Centro de Microscopía, Caracterización y Microanálisis de la Universidad de Australia Occidental (Perth). El revestimiento de carbono fue removido y reemplazado por un recubrimiento en oro para proceder a los análisis U-Pb SHRIMP. Los análisis de U-Pb mediante microsonda iónica de alta resolución y sensibilidad (SHRIMP II) fueron llevados a cabo en la Universidad Curtin (Perth) en cuatro sesiones usando un tamaño de spot analítico circular de 20 a $25 \mu \mathrm{m}$ de diámetro. Para varios granos metamícticos, el tamaño del spot analítico fue reducido a 10 o $15 \mu \mathrm{m}$.

Los análisis individuales están compuestos por nueve mediciones para circón $\left({ }^{196} \mathrm{Zr}_{2} \mathrm{O},{ }^{204} \mathrm{~Pb}\right.$, background, ${ }^{206} \mathrm{~Pb},{ }^{207} \mathrm{~Pb},{ }^{208} \mathrm{~Pb},{ }^{238} \mathrm{U},{ }^{248} \mathrm{ThO}$, $\left.{ }^{254} \mathrm{UO}\right)$, repetidas en cinco escaneos. Para identificar la posición del pico de masa ${ }^{204} \mathrm{~Pb}$ se utilizaron los estándares de circón D23 y vidrio NBS611, en tanto que la calibración del contenido de U y el cociente $\mathrm{Pb} / \mathrm{U}$ se hicieron utilizando el estándar de circón BR266 (559 Ma, 903 ppm U; Stern, 2001). Los datos fueron reducidos utilizando el programa SQUID@ 1.03 (Ludwig, 2001), y las edades se calcularon usando el programa Isoplot $\odot 3.0$ (Ludwig, 2003). Las edades presentadas son edades de concordia ${ }^{206} \mathrm{~Pb} /{ }^{238} \mathrm{U}$ con incertidumbre expresada como $2 \sigma$, mientras que en los análisis individuales está expresada como $1 \sigma$ " (Tabla 1).

\section{Resultados y evaluación}

Se presentan los resultados del Sienogranito Chacharramendi y la Riolita Lihue Calel (Tabla 1).

\section{Sienogranito Chacharramendi (MG88): Pérmico superior (Lopingiano)}

Se analizaron ocho cristales de circón, pero sólo seis se usaron para el cálculo de la edad. Los análisis de los granos 12-1 y 16-1 son altamente discordantes ( $52 \%$ y $67 \%$ ). Los circones son magmáticos, con un contenido promedio de U de $531 \mathrm{ppm}$, y promedio Th/U de 1.15. Un ejemplo de circón datado se ve en Figura 2a (grano elongado, datado $254 \pm 3$ $\mathrm{Ma}$ ). Todos los granos tienen edades con el mismo rango de precisión, agrupándose en la edad ${ }^{206} \mathrm{~Pb} /{ }^{238} \mathrm{U}$ versus ${ }^{207} \mathrm{~Pb} /{ }^{206} \mathrm{~Pb}$ de $254.73 \pm 1.3 \mathrm{Ma}$ $(\mathrm{MSWD}=0.31 ; 2 \sigma)$ (concordia inversa o Tera-Wasserburg, Figura $2 \mathrm{~b})$.

Tabla 1. Datos U-Pb SHRIMP de la Riolita Lihué Calel y el Sienogranito Chacharramendi.

\begin{tabular}{|c|c|c|c|c|c|c|c|c|c|c|c|c|c|c|c|c|c|}
\hline Spot & $\begin{array}{l}{ }^{238} \mathbf{U} \\
\mathrm{ppm}\end{array}$ & $\begin{array}{l}{ }^{232} \mathrm{Th} \\
\mathrm{ppm}\end{array}$ & $\begin{array}{c}{ }^{232} \mathrm{Th} /{ }^{238} \mathrm{U} \\
\text { ratio }\end{array}$ & $\begin{array}{l}{ }^{206} \mathrm{~Pb} \\
\mathrm{ppm}\end{array}$ & $\begin{array}{l}{ }^{207} \mathbf{P b} \\
\mathrm{ppm}\end{array}$ & $\begin{array}{c}{ }^{206} \mathrm{~Pb} \\
\text { común } \\
\%\end{array}$ & $\begin{array}{c}{ }^{238} \mathbf{P b} /{ }^{206} \mathrm{~Pb} \\
\text { ratio }\end{array}$ & $\%$ err & $\begin{array}{c}{ }^{207} \mathbf{P b} /{ }^{206} \mathbf{P b} \\
\text { ratio }\end{array}$ & $\%$ err & $\begin{array}{c}{ }^{208} \mathrm{~Pb} /{ }^{232} \mathrm{Th} \\
\text { ratio }\end{array}$ & $\%$ err & $\begin{array}{c}{ }^{206} \mathbf{P b} /{ }^{238} \mathbf{U} \\
\text { age }\end{array}$ & $1 \mathrm{~s}$ err & $\begin{array}{c}{ }^{207} \mathbf{P b} /{ }^{206} \mathbf{P b} \\
\text { age }\end{array}$ & $1 \mathrm{~s}$ err & $\begin{array}{c}\text { Disc. } \\
\%\end{array}$ \\
\hline \multicolumn{18}{|c|}{ GA119, Riolita Lihue Calel } \\
\hline B.4-1 & 177 & 252 & 1.47 & 5.8 & 0.30 & 0.47 & 26.3826 & 1.88 & 0.05182 & 9.87 & 0.0120 & 3.67 & 239.8 & 4.4 & 277 & 226 & 14 \\
\hline B.5-1 & 117 & 193 & 1.71 & 4.0 & 0.16 & 4.88 & 26.3848 & 2.21 & 0.03927 & 33.37 & 0.0113 & 6.92 & 239.8 & 5.2 & $-399^{*}$ & 870 & $160^{*}$ \\
\hline B.5-2 & 126 & 210 & 1.72 & 4.1 & 0.22 & 1.59 & 26.5326 & 2.03 & 0.05343 & 11.74 & 0.0113 & 4.02 & 238.5 & 4.7 & 347 & 266 & 31 \\
\hline B.9-1 & 178 & 269 & 1.56 & 5.9 & 0.23 & 2.60 & 26.5629 & 1.50 & 0.03937 & 18.09 & 0.0105 & 4.03 & 238.2 & 3.5 & $-392^{*}$ & 471 & $161^{*}$ \\
\hline B.11-2 & 40 & 59 & 1.54 & 1.5 & 0.04 & 11.48 & 26.3001 & 3.92 & 0.02840 & 102.63 & 0.0101 & 18.51 & 240.6 & 9.3 & $-1331^{\star}$ & 3280 & $118^{*}$ \\
\hline B.12-1 & 849 & 445 & 0.54 & 27.9 & 1.31 & 2.21 & 26.7335 & 1.18 & 0.04677 & 6.52 & 0.0105 & 5.89 & 236.7 & 2.7 & 38 & 156 & 84 \\
\hline \multicolumn{18}{|c|}{ MG88, Sienogranito Chacharramendi } \\
\hline B.6-1 & 443 & 569 & 1.33 & 15.4 & 0.79 & 0.55 & 24.7897 & 1.42 & 0.05135 & 4.60 & 0.0122 & 2.01 & 254.9 & 3.6 & 256 & 106 & 1 \\
\hline B.12-1 & 664 & 576 & 0.90 & 22.3 & 1.28 & 0.91 & 25.7682 & 1.22 & 0.05741 & 3.65 & 0.0101 & 2.98 & 245.4 & 2.9 & 507 & 80 & 52 \\
\hline B.13-1 & 1332 & 1499 & 1.16 & 46.2 & 2.36 & 0.56 & 24.8847 & 1.12 & 0.05103 & 2.70 & 0.0120 & 2.02 & 254.0 & 2.8 & 242 & 62 & -5 \\
\hline B.16-1 & 413 & 365 & 0.91 & 13.8 & 0.89 & -0.21 & 25.6658 & 1.35 & 0.06421 & 3.57 & 0.0142 & 1.80 & 246.4 & 3.3 & 749 & 75 & 67 \\
\hline
\end{tabular}

Todos los cocientes y edades están corregidos por plomo común usando la cantidad medida de $204 \mathrm{~Pb}$. Edades $207 \mathrm{~Pb} / 206 \mathrm{~Pb}$ negativas y discordancia mayor que $100 \%$ (marcada con ${ }^{*}$ ) son datos espurios derivados de contenido de $207 \mathrm{~Pb}$ extremadamente bajo $(>0.25 \mathrm{ppm})$. 

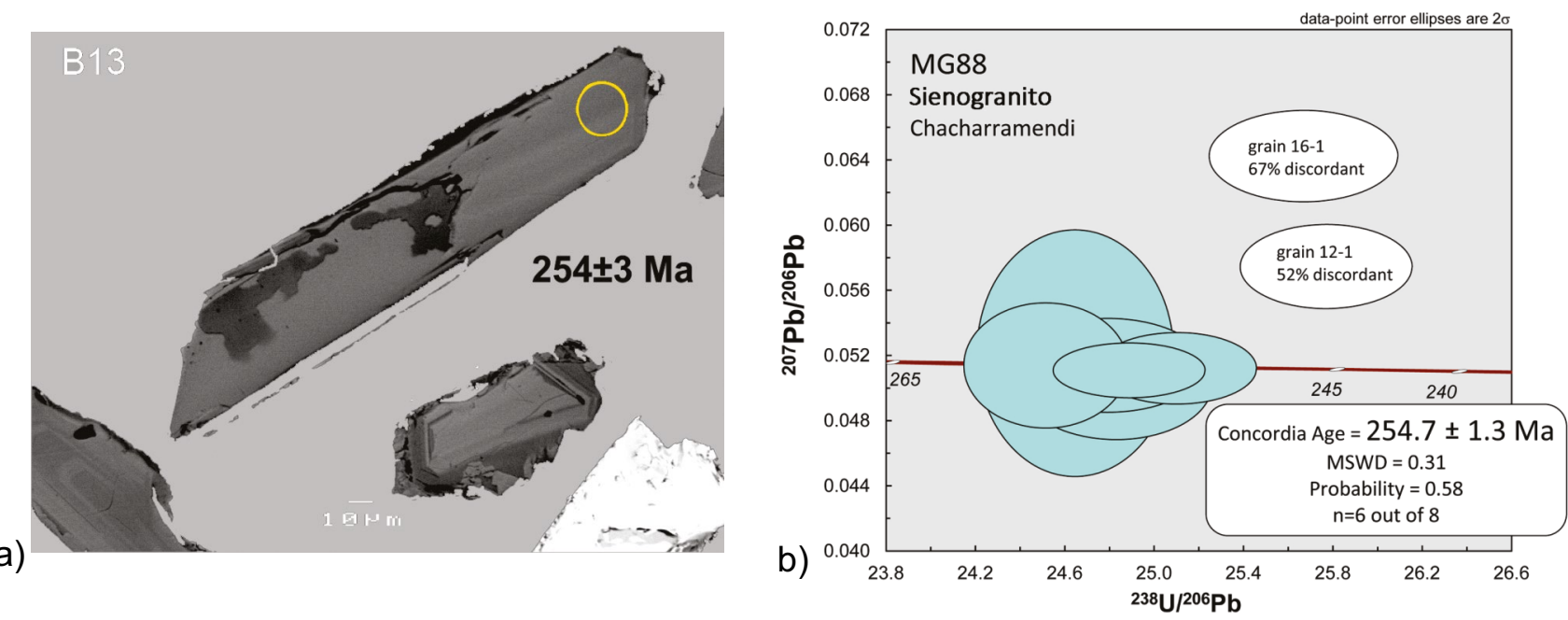

Figura 2. Resultados de la datación U-Pb SHRIMP en circones del Sienogranito Chacharramendi (muestra MG88). a) Imagen de electrones retrodifundidos de uno de los circones analizados; b) Diagrama de concordia inversa $\left({ }^{207} \mathrm{~Pb} /{ }^{206} \mathrm{~Pb} v s .{ }^{206} \mathrm{~Pb} /{ }^{238} \mathrm{U}\right)$ para circones magmáticos de la muestra analizada; la edad de concordia inversa es $254.7 \pm 1.3 \mathrm{Ma}$.

Esta edad corresponde al final del Pérmico, y es considerada como la edad de cristalización del cuerpo granítico.

\section{Riolita Lihue Calel (GA119): Triásico Medio}

Se analizaron nueve cristales de circón. Todos los granos son magmáticos, con un contenido promedio de U de 383 ppm, y promedio Th/U de 1.41 (Tabla 1). Un ejemplo de circón datado se ve en la Figura 3a. Todos los circones son pobres en $\mathrm{U}$ y muy pobres en ${ }^{207} \mathrm{~Pb}$ (el promedio llega sólo a $0.33 \mathrm{ppm}$ ), debido a lo cual, los valores de las edades ${ }^{207} \mathrm{~Pb} /{ }^{206} \mathrm{~Pb}$ y de concordancia no tienen significado. La edad calculada está centrada en las relaciones ${ }^{238} \mathrm{U} /{ }^{206} \mathrm{~Pb}$. Todos los granos tienen edades con el mismo grado de precisión, excepto el grano 11-2 que tiene un alto contenido de $\mathrm{Pb}$ común (12.24\%) y no fue usado en el cálculo de la edad. La edad obtenida con 8 de los 9 análisis es $239 \pm 1.5 \mathrm{Ma}$
$(\mathrm{MSWD}=0.92 ; 2 \sigma)($ Figura $3 \mathrm{~b})$-Triásico Medio (Ladiniano) $-\mathrm{y}$ es considerada como la edad de cristalización de la unidad riolítica.

\section{ISÓTOPOS DE Hf}

\section{Metodología}

Los análisis isotópicos de Lu-Hf en circones se llevaron a cabo en el GEMOC (Geochemical Evolution and Metallogeny of Continents, Macquarie University, Sydney) utilizando una microsonda de ablación laser marca New Wave/Merchantek UP213, acoplada a un espectrómetro de masas de tipo multicolector con plasma de acoplamiento inductivo marca Nu Plasma (Multi-Collector Inductively Coupled Plasma Mass Spectrometry, MC-ICP-MS). Las condiciones de funcionamiento incluyen un haz de $\pm 55 \mu \mathrm{m}$ de diámetro, frecuencia a)

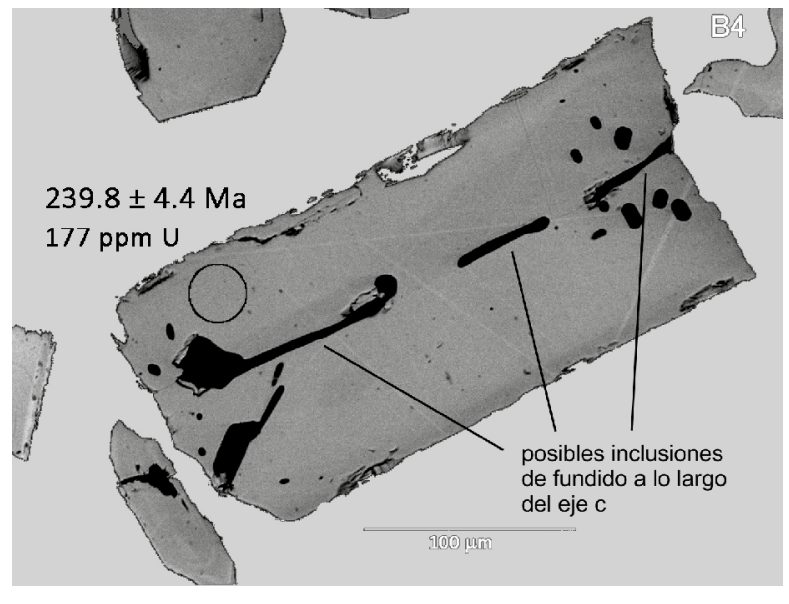

Figura 3. Resultados de la datación U-Pb SHRIMP en circones de la Riolita Lihue Calel (muestra GA119). a) Imagen de electrones retrodifundidos de uno de los circones analizados; b) Diagrama de concordia inversa $\left({ }^{207} \mathrm{~Pb} /{ }^{206} \mathrm{~Pb} v s .{ }^{206} \mathrm{~Pb} /{ }^{238} \mathrm{U}\right)$ para circones magmáticos de la muestra analizada; la edad de concordia inversa es $239.3 \pm 1.5 \mathrm{Ma}$ (la edad promedio es $239.0 \pm 1.9 \mathrm{Ma}$ ).

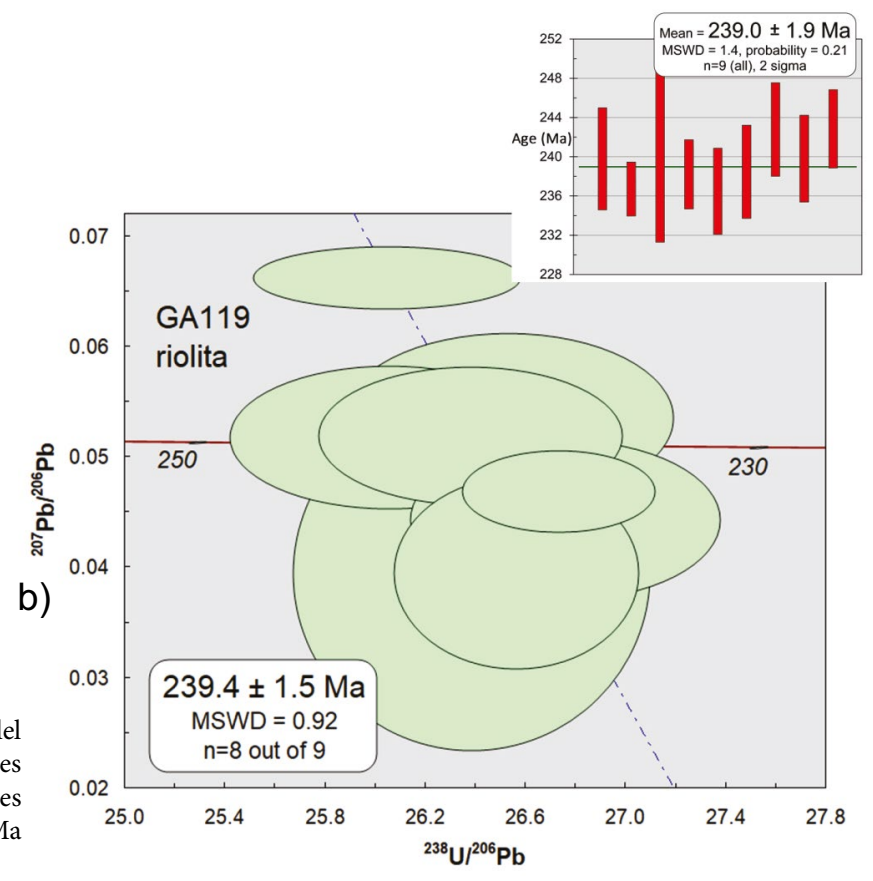


de repetición de $5 \mathrm{~Hz}$, con una energía de $\sim 0.4-0.8 \mathrm{~mJ}$. Los tiempos de ablación fueron de 100-120 segundos, resultando en hoyos de $40-60 \mu \mathrm{m}$ de profundidad. La interferencia isobárica de ${ }^{176} \mathrm{Yb}$ en ${ }^{176} \mathrm{Hf}$ fue corregida midiendo el isótopo ${ }^{172} \mathrm{Yb}$ libre de interferencia, y luego usando el cociente ${ }^{176} \mathrm{Yb} /{ }^{172} \mathrm{Yb}$ para calcular la intensidad del ${ }^{176} \mathrm{Yb}$ libre de interferencia. El valor apropiado de ${ }^{176} \mathrm{Yb} /{ }^{172} \mathrm{Yb}$ fue determinado analizando el stardard de Hf JMC475 (Wiedenbeck et al., 1995), al cual se fueron adicionando sucesivamente varias cantidades de $\mathrm{Yb}$. Debido a que la relación ${ }^{176} \mathrm{Lu} /{ }^{177} \mathrm{Hf}$ en circón es extremadamente baja (normalmente $<0.002$ ), la interferencia isobárica de ${ }^{176} \mathrm{Lu}$ en ${ }^{176} \mathrm{Hf}$ es despreciable (Iizuka and Hirata, 2005). El material de referencia de circón Mud Tank (MT), que tiene un cociente promedio de ${ }^{176} \mathrm{Hf} /{ }^{177} \mathrm{Hf}$ de $0.282522 \pm 42(2 \sigma)$ (Griffin et al., 2007), fue utilizado para medir la precisión de los resultados. El MT analizado en este estudio estuvo dentro del rango reportado previamente $(0.282531 \pm 40 ; n=14)$. El standard de circón Temora-2 también fue utilizado para monitorear la eficiencia de la corrección de $\mathrm{Yb}$. El promedio medido de ${ }^{176} \mathrm{Hf} /{ }^{177} \mathrm{Hf}$ de Temora2 para este estudio es $0.282679 \pm 19(n=3)$, el cual está dentro del rango de valores previamente reportados $(0.282687 \pm 24$; Hawkesworth y Kemp, 2006). Temora-2 tiene cocientes ${ }^{176} \mathrm{Yb} /{ }^{177} \mathrm{Hf}$ típicos de alrededor de 0.04, apenas más altos que los cocientes de ${ }^{176} \mathrm{Yb} /{ }^{177} \mathrm{Hf}$ promedio en este estudio (0.034). Mayores detalles de las técnicas analíticas, precisión y exactitud son descriptos por Griffin et al. $(2000,2004)$.

Los valores iniciales de ${ }^{176} \mathrm{Hf} /{ }^{177} \mathrm{Hf}$ fueron calculados utilizando cocientes medidos de ${ }^{176} \mathrm{Lu} /{ }^{177} \mathrm{Hf}$, con una incertidumbre típica en análisis individuales de ${ }^{176} \mathrm{Lu} /{ }^{177} \mathrm{Hf}$ de $1-2 \%(2 \sigma)$. Tal error refleja tanto los errores analíticos como la variación intragrano de Lu/Hf típicamente observada en circones. Para el cálculo de los valores $\varepsilon_{\mathrm{Hf}}$ se utilizaron la constante de decaimiento de ${ }^{176} \mathrm{Lu}$ de Scherer et al. (2001) $\left(1.865 \times 10^{-11}\right)$ y los valores condríticos (CHUR) de Blichert-Toft y Albarède (1997). Para el cálculo de las edades modelo $\mathrm{Hf}\left(T_{\mathrm{DM}}\right)$ en un paso ("single-stage model ages") se empleó un modelo de evolución del manto empobrecido calculado a partir de valores actuales de ${ }^{176} \mathrm{Hf} /{ }^{177} \mathrm{Hf}$ $=0.28325$ (Griffin et al., 2000, 2004). Las edades modelo de $\mathrm{Hf}\left(T_{\mathrm{DM}}\right)$ en un paso ("single-stage model ages"), las cuales son calculadas usando las relaciones ${ }^{176} \mathrm{Hf} /{ }^{177} \mathrm{Hf}$ y ${ }^{176} \mathrm{Lu} /{ }^{177} \mathrm{Hf}$ medidas en el circón, proporcionan sólo una edad mínima para la fuente del magma del cual el circón ha cristalizado. Por lo tanto, también se ha calculado, para cada circón, una "edad modelo cortical" $\left(T_{\mathrm{DM}}{ }^{\mathrm{C}}\right)$ (edades modelo en dos pasos), la cual asume que la fuente del magma fue producida de una corteza continental promedio con una relación ${ }^{176} \mathrm{Lu} /{ }^{177} \mathrm{Hf}$ de 0.015 (Griffin et al., 2000, 2002) que fue originalmente derivada del manto empobrecido.
El cálculo de los valores $\varepsilon_{\mathrm{Hf}(\mathrm{t})}$ está basado en edades U-Pb SHRIMP en circones y en los valores condríticos (CHUR) de ${ }^{176} \mathrm{Hf} /{ }^{177} \mathrm{Hf}=0.282772$ $y{ }^{176} \mathrm{Lu} /{ }^{177} \mathrm{Hf}=0.0332$ (Blichert-Toft y Albarède, 1997).

\section{Resultados y evaluación}

Sienogranito Chacharramendi (MG88)

Se realizaron seis determinaciones de isótopos de Hf en granos datados de circón del Sienogranito Chacharramendi (Tabla 2). Los cocientes ${ }^{176} \mathrm{Hf} /{ }^{177} \mathrm{Hf}$ de todos los cristales de circón medidos son similares y los datos produjeron valores negativos $\varepsilon_{\mathrm{Hf}}$ (promedio -5.68). La edad modelo Lu-Hf de los circones es $1.64 \mathrm{Ga}$ (Figura 4). Esto significa que el sienogranito aquí analizado fue cristalizado a partir de un fundido con importante contribución de corteza statheriana tardía (1.64 Ga). Esa edad modelo también puede ser un promedio de las edades de una corteza compleja con rocas más antiguas y más jóvenes que $1.64 \mathrm{Ga}$.

\section{Riolita Lihue Calel (GA119)}

Se realizaron cuatro determinaciones de isótopos de Hf en granos de circón de la Riolita Lihue Calel. Los cocientes ${ }^{176} \mathrm{Hf} /{ }^{177} \mathrm{Hf}$ de todos los cristales de circón medidos son similares y los datos produjeron valores negativos $\varepsilon_{\mathrm{Hf}}$ (promedio -5.56; Tabla 2). La edad modelo Lu-Hf promedio de los circones es $1.64 \mathrm{Ga}$ (Figura 5). Esto significa que la riolita aquí analizada fue cristalizada a partir de un fundido con importante contribución de corteza statheriana tardía $(1.64 \mathrm{Ga})$. La coincidencia entre las dos edades modelo de las dos muestras (sienogranito y riolita) indica que es más probable la presencia de una corteza statheriana y que las edades modelo con 1.64 Ga no son el resultado de la fusión de rocas con diferentes edades.

\section{GEOQUÍMICA}

Los análisis químicos de roca total se efectuaron en Activation Laboratories Ltd., Ontario, Canadá. Los elementos mayores se analizaron mediante el método de fusión con metaborato/tetraborato de litio - ICP. La precisión y exactitud es generalmente superior al $2 \%$ (relativo). Los elementos traza y los elementos del grupo de tierras raras (REE) se analizaron mediante un ICP-MS. La precisión y exactitud es generalmente superior al $\pm 6 \%$. El contenido en elementos mayoritarios y traza de rocas seleccionadas del CMPT-LP se presentan en la Tabla 3. En la descripción que sigue se hace referencia a estos valores así como a otros obtenidos por otros autores.

Tabla 2. Composición isotópica de Hf de circones de la Riolita Lihué Calel y el Sienogranito Chacharramendi.

\begin{tabular}{|c|c|c|c|c|c|c|c|c|}
\hline Spot & $\begin{array}{l}{ }^{206} \mathrm{~Pb} /{ }^{238} \mathrm{U} \\
\text { edad (Ma) }\end{array}$ & ${ }^{176} \mathbf{Y b} /{ }^{177} \mathbf{H f}$ & ${ }^{176} \mathrm{Lu} /{ }^{177} \mathrm{Hf}$ & ${ }^{176} \mathbf{H f} /{ }^{177} \mathbf{H f}$ & $\begin{array}{l}\text { error } \\
1 \text { sigma }\end{array}$ & $\varepsilon_{\mathrm{Hf}}$ & $\begin{array}{c}\text { error } \\
1 \text { sigma }\end{array}$ & $\begin{array}{c}\mathrm{T}_{\mathrm{DM}}{ }^{\mathrm{C}} \\
\text { cortical }\end{array}$ \\
\hline \multicolumn{9}{|c|}{ GA119, Riolita Lihue Calel } \\
\hline B.1-1 & 243 & 0.0370055 & 0.00081711 & 0.282475 & 0.0000067 & -5.30 & 0.2345 & 1.61 \\
\hline B. $4-1$ & 240 & 0.0429246 & 0.00094219 & 0.282459 & 0.0000091 & -5.95 & 0.3185 & 1.65 \\
\hline B.5-2 & 238 & 0.0307602 & 0.00075895 & 0.282488 & 0.000023 & -4.93 & 0.805 & 1.58 \\
\hline B.8-1 & 243 & 0.0583882 & 0.00120825 & 0.282455 & 0.0000061 & -6.07 & 0.2135 & 1.66 \\
\hline \multicolumn{9}{|c|}{ MG88, Sienogranito Chacharramendi } \\
\hline B.6-1 & 254.9 & 0.11968 & 0.002289 & 0.282456 & 0.000015 & -5.97 & 0.525 & 1.664 \\
\hline B.6-2 & 251.9 & 0.08265 & 0.001612 & 0.282466 & 0.000011 & -5.57 & 0.385 & 1.636 \\
\hline B.7-1 & 256.4 & 0.04529 & 0.000827 & 0.282458 & 0.000015 & -5.62 & 0.525 & 1.643 \\
\hline B.10-1 & 257.8 & 0.03986 & 0.000814 & 0.282474 & 0.000013 & -5.02 & 0.455 & 1.606 \\
\hline B.13-1 & 254.0 & 0.05247 & 0.001058 & 0.282462 & 0.000010 & -5.57 & 0.3465 & 1.638 \\
\hline B.16-1.5 & 243.4 & 0.06003 & 0.001193 & 0.282447 & 0.000010 & -6.35 & 0.350 & 1.678 \\
\hline
\end{tabular}




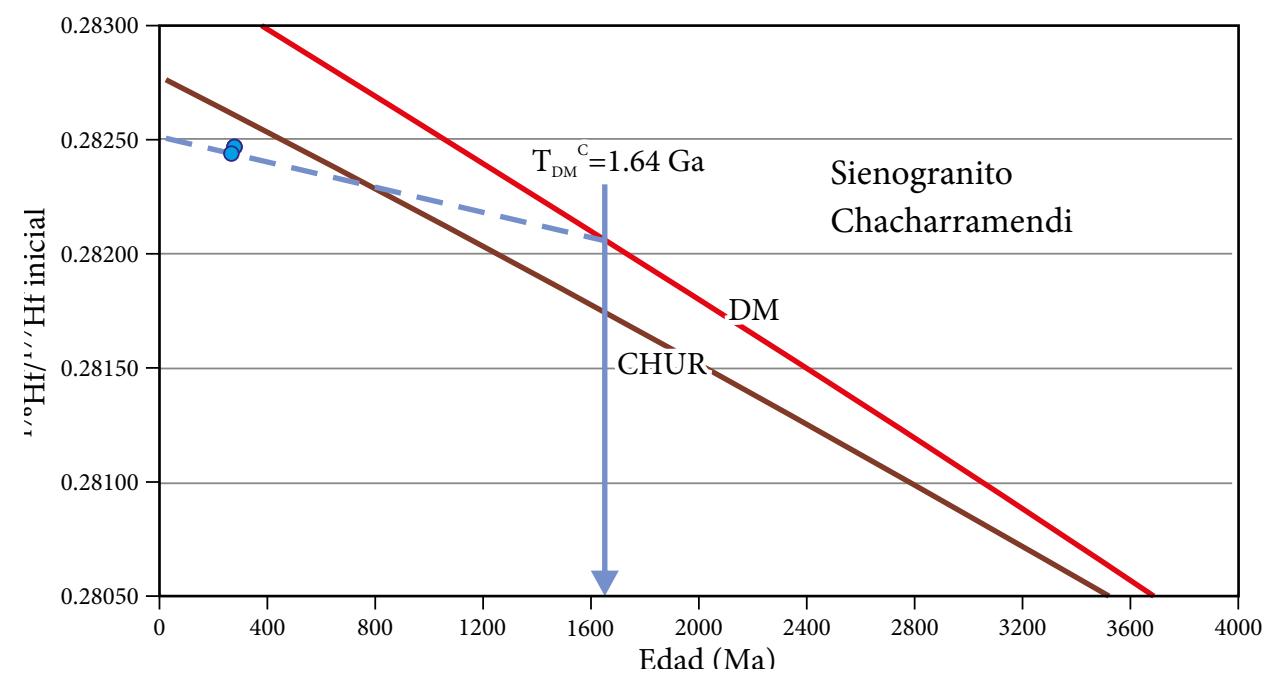

Figura 4. Diagrama ${ }^{176} \mathrm{Hf} /{ }^{177} \mathrm{Hf}$ versus edad de seis circones datados de la muestra MG88 (Sienogranito Chacharramendi). La edad modelo cortical, $\mathrm{T}(\mathrm{DM})$ C, $_{\text {, relativa }}$ al manto empobrecido es statheriana tardía $(1.64 \mathrm{Ga})$. La pendiente de la línea de guiones se calculó con un valor de 0.015 para el cociente ${ }^{176} \mathrm{Lu} /{ }^{177} \mathrm{Hf}$. DM: Manto empobrecido; CHUR: reservorio condrítico uniforme.

El CMPT-LP está constituido predominantemente por volcanitas riolíticas $\left(\mathrm{SiO}_{2}\right.$ entre 70 y $\left.78 \%\right)$ y sienogranitos $\left(\mathrm{SiO}_{2}\right.$ entre 74 y $\left.78 \%\right)$. De manera subordinada hay traquitas $\left(\mathrm{SiO}_{2}: 61\right.$ a $\left.66 \%\right)$, bostonitas $\left(\mathrm{SiO}_{2}\right.$ : $55 \%)$, sienitas $\left(\mathrm{SiO}_{2}: 57\right.$ a $\left.62 \%\right)$ y trondhjemitas $\left(\mathrm{SiO}_{2}: 71\right.$ a $\left.75 \%\right)$ (Nota: se toman las trondhjemitas del Plutón Las Matras, originalmente datadas como mesoproterozoicas por Sato et al., 2000, debido a su reciente asignación al Pérmico; Chernicoff et al., 2016b). Entre las variedades alcalinas se clasifican las sienitas de López Lecube y Estancia El Trabajo, la bostonita de Paso del Bote y la traquita comendítica de Puesto Garro, y en parte las riolitas y granitos $\left(\mathrm{Na}_{2} \mathrm{O}+\mathrm{K}_{2} \mathrm{O}>8\right)$. En su conjunto, las rocas del CMPT-LP son del tipo alto potasio $\left(\mathrm{K}_{2} \mathrm{O} / \mathrm{Na}_{2} \mathrm{O}>1\right)$ excepto las trondhjemitas $\left(\mathrm{K}_{2} \mathrm{O} / \mathrm{Na}_{2} \mathrm{O} \sim 0.6-0.7\right)$. En el diagrama $\mathrm{A} / \mathrm{CNK} v s$. A/NK de Maniar y Piccoli (1989) -no mostrado- las rocas intermedias se agrupan en el campo metaluminoso, en tanto las más ácidas lo hacen cercanas al límite de los campos metaluminoso e hiperaluminoso, lo que se refleja en el contenido de corindón normativo en las riolitas ( $c f$. Sruoga y Llambias, 1992). El contenido de $\mathrm{TiO}_{2}, \mathrm{Al}_{2} \mathrm{O}_{3}, \mathrm{Fe}_{2} \mathrm{O}_{3} \mathrm{t}, \mathrm{MnO}$,
$\mathrm{MgO}$ y CaO muestra un decrecimiento gradual con el incremento de $\mathrm{SiO}_{2}$, en tanto $\mathrm{Na}_{2} \mathrm{O}$ y K $\mathrm{K}_{2} \mathrm{O}$ no presentan correlación con $\mathrm{SiO}_{2}$.

Las rocas, analizadas por grupo litológico, en general muestran un rango de variación de elementos traza restringido, de entre 1 y 3 veces (e.g., REE en granito entre 100 y 200 ppm, REE en sienitas entre 300 y 600 ppm). En contraste, algunos elementos muestran una gran variación, de entre 20 y 40 veces, tales como Ba (entre 50 y 1400 ppm en riolitas y entre 40 y 1400 ppm en granitos), Co (entre 2 y 30 ppm en riolitas y entre 1 y 160 ppm en granitos) y Cr (entre 4 y 75 ppm en riolitas y entre 10 y 200 ppm en granitos).

El contenido de REE normalizado a basaltos de islas oceánicas (OIB) muestra un patrón subhorizontal, con presencia de moderadas anomalías negativas de Eu en granitos y riolitas y leve enriquecimiento en REE pesadas en las trondhjemitas (Figura 6a). En los diagramas de variación multielemental normalizados al condrito de Thompson (1982), entre los elementos litófilos de radio iónico grande (LILE), el $\mathrm{Ba}$ y el Sr presentan anomalías negativas pronunciadas, en tanto que

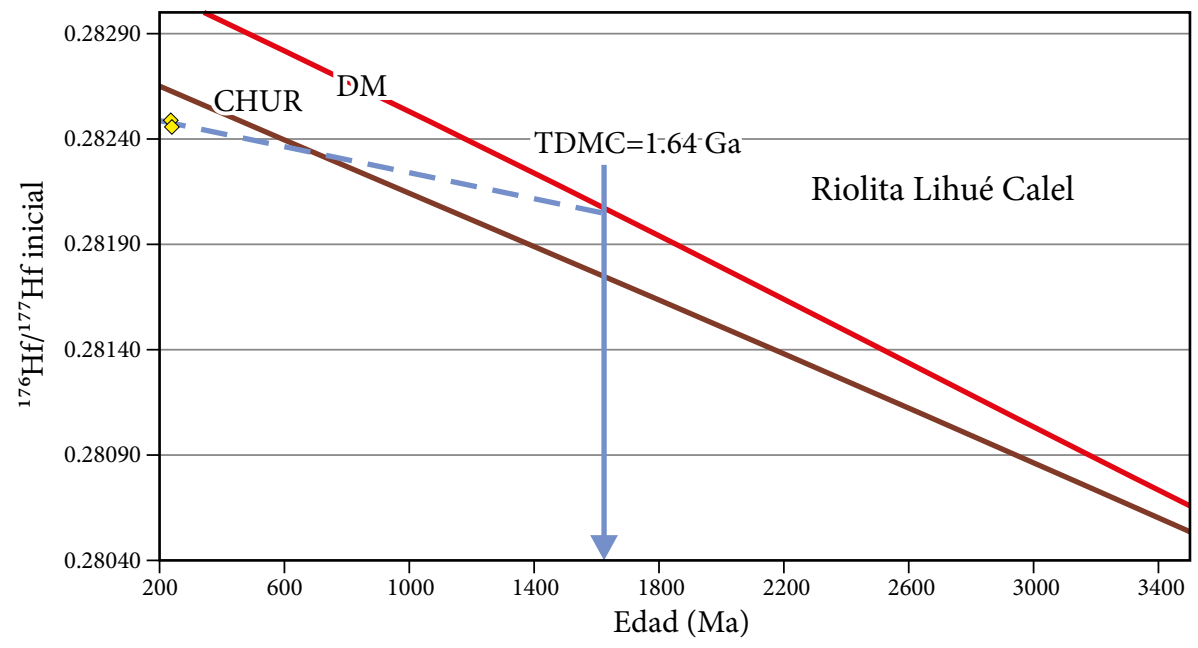

Figura 5. Diagrama ${ }^{176} \mathrm{Hf} /{ }^{177} \mathrm{Hf}$ versus edad de cuatro circones datados de la muestra GA119 (Riolita Lihue Calel). La edad modelo (cortical) del manto empobrecido es statheriana tardía $(1.64 \mathrm{Ga})$. La pendiente de la línea de guiones se calculó con un valor de 0.015 para el cociente ${ }^{176} \mathrm{Lu} /{ }^{177} \mathrm{Hf}$. DM: Manto empobrecido; CHUR: reservorio condrítico uniforme. 
Tabla 3. Concentración de elementos mayores y traza en rocas del Corredor Magmático Intracratónico Pérmico-Triásico de La Pampa (CMPT-LP).

\begin{tabular}{|c|c|c|c|c|}
\hline Localidad & $\begin{array}{l}\text { Paso del } \\
\text { Bote }\end{array}$ & $\begin{array}{c}\text { Chacharra- } \\
\text { mendi }\end{array}$ & $\begin{array}{c}\text { Estancia } \\
\text { El Trabajo }\end{array}$ & Las Matras \\
\hline Muestra & PB82 & MG156 & CHZ71 & MG160 \\
\hline Litología & Bostonita & Granito & Sienita & Trondhjemita \\
\hline Latitud & -38.02315 & -37.32143 & -38.22258 & -36.78333 \\
\hline Longitud & -65.82467 & -65.71778 & -65.68762 & -67.12300 \\
\hline \multicolumn{5}{|l|}{ (\% en peso) } \\
\hline $\mathrm{SiO}_{2}$ & 55.03 & 74.11 & 58.7 & 71.7 \\
\hline $\mathrm{TiO}_{2}$ & 0.836 & 0.14 & 0.997 & 0.28 \\
\hline $\mathrm{Al}_{2} \mathrm{O}_{3}$ & 14.82 & 13.29 & 12.55 & 13.08 \\
\hline $\mathrm{Fe}_{2} \mathrm{O}_{3} \mathrm{t}$ & 6.06 & 1.87 & 6.07 & 3.08 \\
\hline $\mathrm{MnO}$ & 0.105 & 0.03 & 0.09 & 0.05 \\
\hline $\mathrm{MgO}$ & 3.29 & 0.1 & 4.11 & 0.44 \\
\hline $\mathrm{CaO}$ & 6.11 & 0.62 & 4.93 & 1.8 \\
\hline $\mathrm{Na}_{2} \mathrm{O}$ & 3.74 & 3.83 & 2.79 & 4.53 \\
\hline $\mathrm{K}_{2} \mathrm{O}$ & 3.34 & 5.32 & 6.96 & 2.9 \\
\hline $\mathrm{P}_{2} \mathrm{O}_{5}$ & 0.39 & 0.02 & 0.98 & 0.07 \\
\hline LOI & 6.85 & 0.8 & 0.87 & 1.1 \\
\hline Total & 100.3 & 99.12 & 99.05 & 99.06 \\
\hline \multicolumn{5}{|l|}{$(p p m)$} \\
\hline $\mathrm{Ba}$ & 1111 & 259.8 & 4505 & 788.6 \\
\hline $\mathrm{Rb}$ & 100 & 268.1 & 162 & 44.2 \\
\hline $\mathrm{Sr}$ & 311 & 99 & 1524 & 177 \\
\hline Cs & 3.4 & 2.8 & 2.7 & 0.3 \\
\hline $\mathrm{Ga}$ & 17 & 21 & 23 & 13 \\
\hline $\mathrm{Ta}$ & 0.7 & 1.2 & 0.8 & $<$ l.d. \\
\hline $\mathrm{Nb}$ & 12 & 28 & 15 & 8 \\
\hline Hf & 4.4 & $<$ l.d. & 9.2 & 2 \\
\hline $\mathrm{Zr}$ & 182 & 122.8 & 335 & 186.4 \\
\hline $\mathrm{Y}$ & 14 & 10.7 & 27 & 28.6 \\
\hline Th & 9.9 & 21.3 & 22.4 & 3.7 \\
\hline $\mathrm{U}$ & 1.7 & 1.15 & 2.7 & 1.35 \\
\hline $\mathrm{Cr}$ & 200 & $<$ l.d. & 90 & $<$ l.d. \\
\hline $\mathrm{Ni}$ & 80 & $<$ l.d. & 70 & $<$ l.d. \\
\hline Co & 30 & 65.7 & 47 & 45.5 \\
\hline $\mathrm{Sc}$ & 13 & 7.6 & 14 & 12.1 \\
\hline $\mathrm{V}$ & 123 & 40 & 164 & 49 \\
\hline $\mathrm{Cu}$ & $<$ l.d. & 5 & 70 & 7 \\
\hline $\mathrm{Pb}$ & 45 & 32 & 56 & 11 \\
\hline $\mathrm{Zn}$ & 70 & 12 & 80 & 45 \\
\hline $\mathrm{La}$ & 42.3 & 34.6 & 132 & 21.5 \\
\hline $\mathrm{Ce}$ & 83.4 & 64.2 & 260 & 49.1 \\
\hline $\operatorname{Pr}$ & 9.17 & 7.4 & 30 & 5.89 \\
\hline $\mathrm{Nd}$ & 33.3 & 24.4 & 119 & 22.9 \\
\hline $\mathrm{Sm}$ & 6 & 3.8 & 19.7 & 5.1 \\
\hline $\mathrm{Eu}$ & 1.43 & 0.71 & 4.47 & 1.1 \\
\hline Gd & 4.7 & 3.24 & 13 & 5.66 \\
\hline $\mathrm{Tb}$ & 0.6 & 0.36 & 1.4 & 0.73 \\
\hline Dy & 2.9 & 1.88 & 5.8 & 4.6 \\
\hline Ho & 0.5 & 0.33 & 1 & 0.95 \\
\hline $\mathrm{Er}$ & 1.6 & 1.1 & 2.4 & 3.36 \\
\hline $\mathrm{Tm}$ & 0.23 & 0.17 & 0.33 & 0.45 \\
\hline $\mathrm{Yb}$ & 1.5 & 1.2 & 2.0 & 2.9 \\
\hline $\mathrm{Lu}$ & 0.21 & 0.2 & 0.28 & 0.49 \\
\hline
\end{tabular}

entre los elementos de alto potencial iónico (HFSE) presentan una marcada depresión en Ti y moderada en $\mathrm{Nb}$, anomalías que son menos pronunciadas en las sienitas (Figura $6 \mathrm{~b}$ ).

En el diagrama $\mathrm{Rb} v s$. $\mathrm{Y}+\mathrm{Nb}$ de Pearce et al. (1984) (Figura 6c) las rocas estudiadas se agrupan en el campo de los granitos de intraplaca. Por otra parte en el diagrama discriminante de granitoides de Whalen et al. (1987) (Figura 6d), la totalidad de las muestras se ubican en el campo de los granitoides del tipo A. En tanto, en el diagrama de $\mathrm{TiO}_{2}$ versus $\mathrm{SiO}_{2}$ de Maniar y Piccoli (1989) (Figura 6e) las muestras se ubican en el campo de rift continental abortado. Estas características contrastan con el ambiente de subducción sugerido por las anomalías negativas de $\mathrm{Ti}$, Ta y $\mathrm{Nb}$ en el diagrama multielemental (Figura 6b). Sin embargo, el ambiente tectónico no es consistente con una subducción activa para la edad y posición del Corredor CMPT-LP analizado, no descartándose entonces una herencia geoquímica a partir de rocas de arco más antiguas (arco famatiniano y/o arco pampeano).

El análisis de la signatura química de los granitos del CMPT-LP es consistente con la caracterización de aquéllos de tipo tipo A de Eby (1990), en los que cocientes de $\mathrm{Y} / \mathrm{Nb}<1.2$ corresponden a fuentes químicamente similares a aquéllas a partir de las cuales se forman los basaltos OIB. En efecto, los cocientes Y/Nb en el CMPT-LP varían entre 0.3 y 0.9 , y por lo tanto las rocas del CMPT-LP también clasifican en el campo $A_{1}$ de Eby (1992) -en contraste con el campo $A_{2}$ del mismo autor, asignado a suites tipo $\mathrm{A}$ con cocientes $\mathrm{Y} / \mathrm{Nb}>1.2$, derivadas de fuentes químicamente similares a basaltos de arco de islas o de margen continental. En la Figura $6 \mathrm{f}$ se muestra como los campos correspondientes a los arcos Choiyoi y Gondwánides se agrupan por separado del campo correspondiente al CMPT-LP, en virtud de sus cocientes $\mathrm{Y} / \mathrm{Nb}>1.2 \mathrm{o}<1.2$, respectivamente.

\section{DISCUSIÓN}

\section{Datos isotópicos y geoquímicos}

Se ha realizado un estudio y revisión de las unidades magmáticas pérmicas-triásicas de la provincia de La Pampa, Argentina -con continuidad en el sur de las provincias de Mendoza y Buenos Airesque integran lo que aquí denominamos el Corredor Magmático Intracratónico Pérmico-Triásico de La Pampa (CMPT-LP; Figura 1). Se dan a conocer nuevas edades U-Pb SHRIMP, composición isotópica de Hf en circones y composición química de algunas unidades integrantes del CMPT-LP.

Las nuevas edades aquí presentadas corresponden al Sienogranito Chacharramendi datado por el método U-Pb SHRIMP en circones, el que arrojó una edad de $254.7 \pm 1.3 \mathrm{Ma}$ (Pérmico Superior, Lopingiano) y una edad modelo Hf de $1640 \mathrm{Ma}$ con un valor $\varepsilon_{\mathrm{Hf}}$ promedio de -5.68. Asimismo, una datación realizada también por el mismo método en circones de la Riolita Lihue Calel arrojó una edad de $239.3 \pm 1.5 \mathrm{Ma}$ (Triásico Medio), y una edad modelo Hf de $1640 \mathrm{Ma}$ con un valor $\varepsilon_{\mathrm{Hf}}$ promedio de -5.56. Esto indica que las dos unidades datadas fueron cristalizadas, dentro del amplio rango permo-triásico, a partir de un fundido que incorporó corteza de edad statheriana tardía, lo cual también es consistente con la ocurrencia de circones heredados statherianos en la traquiandesita Cerro Centinela $(1871 \pm 13 \mathrm{Ma}$; Barrionuevo et al., 2013), del sector noroccidental del CMPT-LP (Figura 1). Tomando también en cuenta la edad modelo Nd de $1483 \mathrm{Ma}$ y valores de $\varepsilon_{\mathrm{Nd}}$ de -5.6 obtenidos para el Granito López Lecube (258 \pm 2 Ma; Pankhurst et al., 2006) ubicado en el extremo sudoriental del CMPT-LP (Figura 1), este corredor magmático en su conjunto se habría formado a partir de un fundido mantélico que incorporó corteza statheriana tardía-calymmiana.

Nuevos datos geoquímicos aquí aportados, analizados junto con 

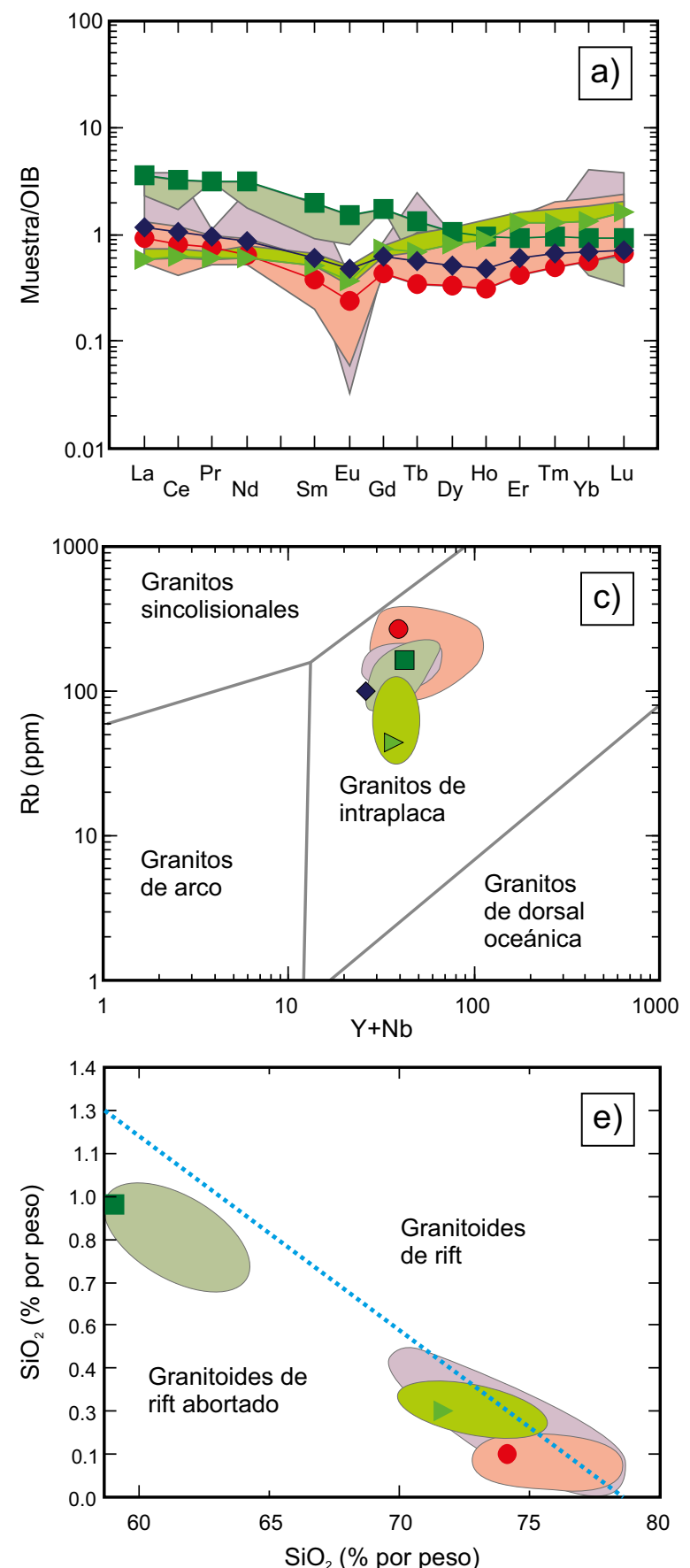

Granito (Algarrobo del Águila, López Lecube y Chacharramendi)

Riolita (Lihue Calel y Cerro Colón) Sienita (López Lecube) y traquita (Puesto Garro)

Trondhjemita (Las Matras)
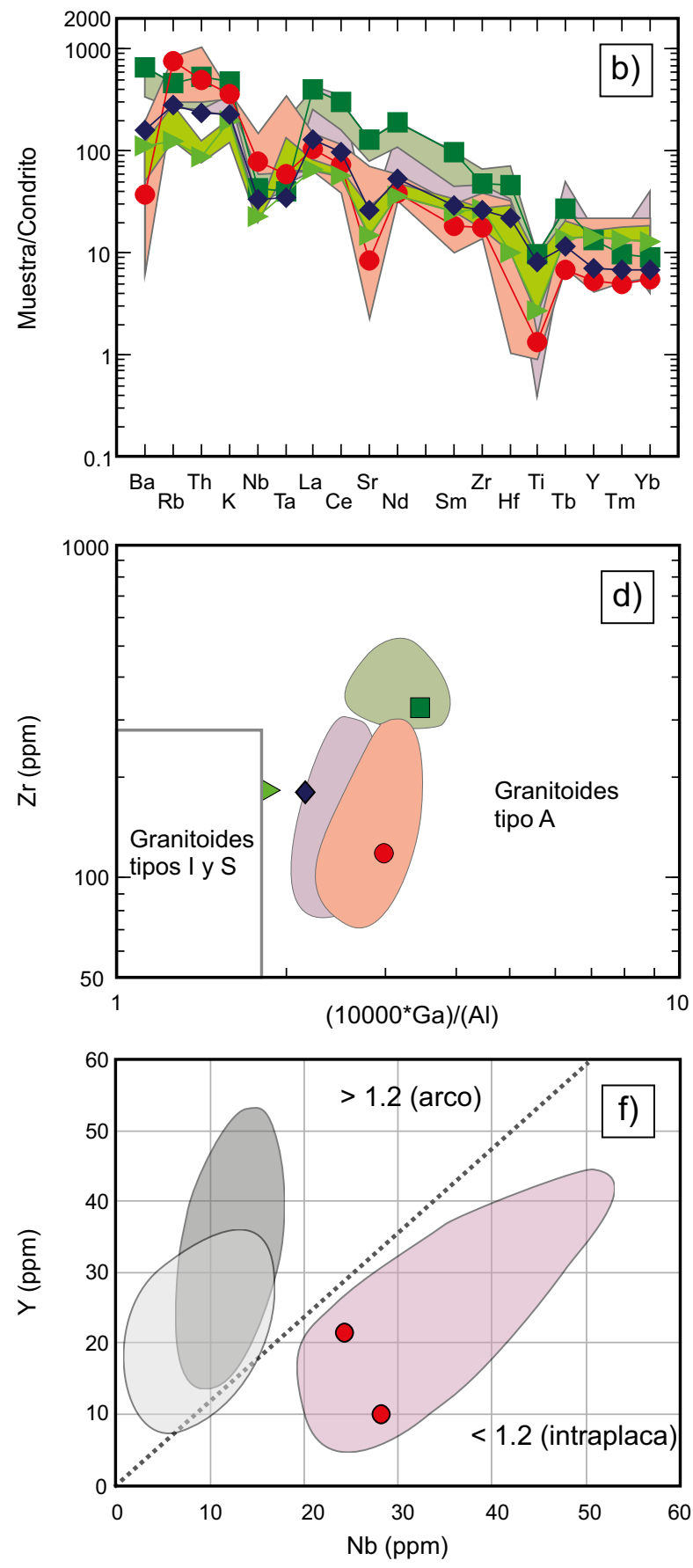

Corredor CMPT-LP

Arco Choiyoi

Arco Gondwánides

Figura 6. Diagramas de elementos traza y de discriminación de ambientes tectónicos para las rocas del Corredor Magmático Intracratónico Pérmico-Triásico de La Pampa (CMPT-LP). a) Diagrama de elementos de las tierras raras normalizado a OIB (Sun, 1980); b) Diagrama de variación multielemental normalizado al condrito de Thompson (1982); c) diagrama geotectónico Rb vs. Y+Nb de Pearce et al. (1984); d) Diagrama de discriminación de granitoides de Whalen et al. (1987); e) Diagrama de $\mathrm{TiO}_{2}$ versus $\mathrm{SiO}_{2}$ de Maniar y Piccoli (1989); f) cociente Y/Nb para los campos del CMPT-LP y de los Arcos Choiyoi y Gondwánides. Referencias para las figuras a-e: Campos de (1) riolitas a partir de Sruoga y Llambías (1992) (riolita Lihuel Calel) y Llambías et al. (2003) (riolita Cerro Colón); (2) granitos a partir de Llambías et al. (2003) (Algarrobo del Aguila y Chacharramendi) y Gregori et al. (2003) (López Lecube); (3) sienitas y traquitas a partir de Gregori et al. (2003) (López Lecube), Dristas et al. (1998) (Puesto Garro); y (4) trondhjemitas a partir de Sato et al. (2000). Referencias para la figura f: datos propios y de Kleiman y Japas (2009), Llambías et al. (2003), Rocher et al. (2015), Sato et al. (2015) y Varela et al. (2015). 
datos geoquímicos previos, denotan la impronta o huella anorogénica -ambiente de intraplaca- del corredor magmático CMPT-LP, destacándose su consistencia con los granitos del tipo $A_{1}$ de Eby (1992) debido a la similitud de sus fuentes con aquéllas a partir de las cuales se forman los basaltos de islas oceánicas ( $\mathrm{Y} / \mathrm{Nb}<1.2)$, contrastante con los cocientes $\mathrm{Y} / \mathrm{Nb}>1.2$ hasta 5 de los granitoides de las dos fajas orogénicas lindantes del CMPT-LP (véase más abajo), i.e., fajas Sanrafaélica (Arco Choiyoi) y Gondwánides Nordpatagónicos (Figura 6f). La pertenencia de las rocas del CMPT-LP mayoritariamente al tipo de alto potasio también es consistente con su ocurrencia en un ambiente tectónico de intraplaca.

Queda claro por lo tanto que (1) los datos isotópicos indican que las fuentes magmáticas del CMPT-LP son predominantemente corticales, si bien datos isotópicos adicionales podrían dar indicios de una mayor heterogeneidad de las fuentes, como ocurre en el magmatismo anorogénico de otras localidades (p. ej., Baldo et al., 2006, 2008), y (2) que los datos geoquímicos del CMPT-LP indican su formación en un ambiente extensional durante el Pérmico tardío-Triásico Medio).

\section{Contexto tectónico del CMPT-LP}

El ambiente anorogénico del CMPT-LP contrasta con aquél de la etapa tardía del Grupo Choiyoi propiamente dicho, que si bien también está asociada a extensión pérmico tardía-triásico temprana, ocurre en el contexto orogénico propio del Arco Magmático Choiyoi (p. ej., Rocher et al., 2015). Asimismo, Hervé et al. (2014) han reportado que, durante esta etapa tardía, los equivalentes plutónicos del Grupo Choiyoi en la Cordillera Frontal de Chile tienen derivación mantélica según lo indican datos isotópicos de $\mathrm{Hf}$ y $\mathrm{O}\left(+2<\varepsilon_{\mathrm{Hf}}<+7\right.$, y $\delta^{18} \mathrm{O}=$ $+4 \%$ ), por tanto, muy distintos a los datos isotópicos del CMPT-LP, siendo esta discrepancia otro indicio para desvincular el CMPT-LP del Grupo Choiyoi. La Tabla 4 esquematiza la subdivisión del magmatismo pérmico-triásico de La Pampa tal como se sugiere en el presente artículo, en el que se deslindan los productos y procesos asociados a la región cratónica, de aquéllos asociados a la región orogénica.

La gran similitud geoquímica e isotópica que hay entre el Granito López Lecube, ubicado en el extremo sudoriental del CMPT-LP y por lo tanto de indudable localización intracratónica (Figura 1), y el resto del CMP-LP refuerza la asignación de este corredor magmático -aún de su sector más cercano al ámbito orogénico pérmico-triásico del Arco Choiyoi- a un ambiente intracratónico.

Por otra parte, el ambiente cratónico en el que se desarrolló el CMPT-LP es consistente con la ausencia de deformación compresiva -Fase Orogénica San Rafael FOSR (Cisuraliano) ausente- en su roca de caja sedimentaria pérmica inferior, i.e., Formación Carapacha (p. ej., Tomezzoli y Melchor, 2002), según el uso de este término en su sentido restrictivo que abarca los afloramientos de conglomerados y areniscas subhorizontales de las sierras de Carapacha Chica y Gould (véase Chernicoff y Zappettini, 2004 y Chernicoff et al., 2008).

El ambiente estable que tuvo la sedimentación pensilvánicapérmica inferior en la cuenca de Carapacha también es observable en el sector más oriental de la cuenca de San Rafael (Limarino y Spalletti, 2006, Spalletti y Limarino, 2017), adyacente y aproximadamente contemporánea a la primera, por lo que probablemente el conjunto debería considerarse como parte de una cuenca intracratónica cuyo inicio extensional antecedería brevemente al corredor magmático CMPT-LP aquí estudiado.

En contraste, más hacia el noroeste, ya en la región orogénica, el sustrato sedimentario del Grupo Choiyoi propiamente dicho (Formación El Imperial, Pensilvánico-Pérmico muy temprano) fue sujeto a la deformación compresiva que ocasionó la FOSR en el segmento principal de la cuenca de San Rafael.

Este modelo es compatible con la serie de cuencas neopaleozoicas que acompañan el orógeno Gondwanides, incluyendo las cuencas de antepaís Karoo, Hesperides y Claromecó (p. ej., Pángaro et al. 2016, y otros autores allí citados), sedimentación que en el área de la provincia de La Pampa en parte pudo haberse superpuesto con los sedimentos intracratónicos de la cuenca de Carapacha-San Rafael oriental.

Es posible que la orientación $c a$. NO-SE en la que se desarrolló el corredor magmático CMPT-LP esté relacionada a la dirección del esfuerzo tensional principal, asociado a la subducción pensilvánicapérmica inferior oblicua ( NO-SE) al margen continental (p. ej., Kleiman y Japas, 2009), propagado desde la región orogénica hacia el área cratónica. La orientación NO-SE de los diques bostoníticos aflorantes en el lecho del río Salado en la proximidad de la localidad de Puelches (Figura 1) es consistente con esta dirección de esfuerzo tensional.

Tabla 4. Subdivisión del magmatismo permo-triásico de La Pampa -región cratónica y región orogénica-, con indicación de los procesos y productos asociados.

\begin{tabular}{cc}
\hline Región orogénica: & Región cratónica: \\
\hline Arco Choiyoi & $\begin{array}{c}\text { Corredor magmático } \\
\end{array}$ \\
& $\begin{array}{c}\text { Intracratónico Permo-Triásico de La Pampa } \\
\text { (CMPT-LP) } \\
\text { (este trabajo) }\end{array}$ \\
\hline
\end{tabular}

Superposición espacial de losproductos de una:

Etapa inicial caracterizada por un contexto compresional, de avance de la placa subductada (overriding plate) hacia la placa en subducción (unterriding plate), i.e., "advancing accretionary orogen", sensu Cawood y Buchan (2007): andesitas-dacitas + granodioritas cal-coalcalinas (p. ej., Sato et al., 2015); derivación principalmente cortical $\left(-6<\varepsilon \mathrm{Hf}<0\right.$ y $\delta^{18} \mathrm{O}=+7 \%$; Hervé et al., 2014),

y de una

Etapa final caracterizada por un contexto extensional causado por el retroceso (rollback) de la placa en subducción (Rocher et al., 2015), i.e., "retreating accretionary orogen", sensu Cawood y Buchan (2007): riolitas + granitos alcalinos (p.ej., Sato et al., 2015). Componentes plutónicos con derivación mantélica $\left(+2<\varepsilon \mathrm{Hf}<+7\right.$ y $\delta^{18} \mathrm{O}=+4 \%$; Hervé et al., 2014),

Leucogranitos de la Formación Zúñiga (incluye Granito Chacharramendi), lavas e ignimbritas andesíticas a riolíticas de las formaciones Centinela y Choique Mahuida ( $p$.ej., Llambías et al., 2003; Sato et al., 2015), y Granito López Lecube (p. ej., Gregori et al., 2003). Derivación cortical (eHf -5.6 a -5.4: este trabajo; eNd -5.6: Pankhurst et al., 2006). 
Ulteriormente, esta dirección pudo haber actuado como anisotropía condicionante de los desplazamientos transcurrentes oblicuos a las cuencas de rift del Cretácico Inferior ubicadas en el deslinde Pampia-cratón del Río de la Plata (Uliana et al., 1989; Zappettini et al., 2015), como ocurre por ejemplo en las cuencas de General Lavalle y Macachín, particionadas en pequeños depocentros desplazados por fallas transcurrentes con dirección NO-SE (p. ej., Webster et al., 2004; de Elorriaga, 2010). El CMPT-LP, que es cortado oblicuamente por este rosario submeridianal de cuencas de rift del Cretácico Inferior, puede ser considerado análogo a este último por su carácter intracratónico y extensional.

\section{Dos fajas orogénicas lindantes del CMPT-LP}

El CMPT-LP se localiza dentro de un área limitada por dos fajas orogénicas Gondwánicas: la Faja Sanrafaélica por el oeste, y la faja Gondwánides Nordpatagónicos por el sur. Aunque mayormente se considera que hay una evolución transicional entre estas dos fajas (Kleiman y Japas, 2009, Pángaro et al., 2016, entre otros), una revisión de sus contextos tectónicos indica que entre las mismas hay un contraste evolutivo que permite separarlas temporal y espacialmente. A saber:

1) Faja Sanrafaélica, de carácter acrecional; la subducción asociada a esta Faja de orientación submeridiana ocurrió entre el Pensilvánico (Pennsylvaniano) y el Pérmico inferior (Cisuraliano), con una polaridad hacia el Este (p. ej., Sato et al., 2015). Hacia el norte del Bloque San Rafael, esta Faja continúa en el noroeste argentino y, aún más al norte, hasta el sector central de Perú (véase sección de Magmatismo Gondwánico).

2) Faja Gondwánides Nordpatagónicos, de carácter colisional; la subducción que precede al evento colisional patagónico tiene polaridad hacia el sur (p. ej., Ramos, 2008). La orientación de esta Faja es aproximadamente este-oeste. En el núcleo orogénico esta colisión está fechada como guadalupiana tardía (ca. $261 \mathrm{Ma}$, Capitaniano; Chernicoff et al., 2013) a lopingiana (Pángaro et al., 2016). Esta Faja continúa en la Faja Plegada del Cabo, en el África meridional, alcanzando las Montañas Pensacola en la Antártida oriental (p. ej., Paton et al., 2016, y referencias allí citadas).

Tradicionalmente, ambas fajas son integradas en la denominación genérica de Gondwánides desde los trabajos pioneros de Keidel (1916) y Du Toit (1927), hasta los más recientes como los de Llambías et al. (2003), Kleiman y Japas (2009), Rocha-Campos et al. (2011), LópezGamundi et al. (2013), Poma et al. (2014), Rocher et al. (2015), Sato et al. (2015), Pángaro et al. (2016), entre otros. Estos trabajos tienen en común que sostienen la existencia de una única faja orogénica paleozoica superior -los Gondwánides-, de la que en esta sección sólo nos ocupamos del segmento que abarca las Fajas Sanrafaélica y de los Gondwánides Nordpatagónicos.

Consideramos que la diferencia que hay entre los estadios evolutivos de estas dos últimas fajas Gondwánicas - p. ej., claramente contrastaste durante el período Guadalupiano a Lopingiano/Triásico Inferior-podría no ser el resultado de un progresivo diacronismo a lo largo de una faja continua - p. ej., Kleiman y Japas (2009): "magmatismo $y$ deformación progresivamente más jóvenes hacia el sudeste, desde la Faja Sanrafaélica hasta los Gondwánides Norpatagónicos"-, sino de una discontinuidad o quiebre espacio-temporal entre estas dos fajas Gondwánicas, discontinuidad que se localiza en la región central, anorogénica, de la provincia de La Pampa, Argentina, que nos ocupa en este trabajo, y que se muestra en la Figura 1.

Sucintamente, el contraste evolutivo en el período Guadalupiano a Lopingiano/Triásico Inferior se evidencia, por ejemplo, en que

a) mientras en la Faja Sanrafaélica (o Arco Magmático Choiyoi) aún continuaba la subducción de la placa paleopacífica bajo el margen continental Gondwánico (p. ej., Charrier et al., 2014 y referencias alli citadas), el retroarco Choiyoi era sometido a extensión (p. ej., Rocher et al., 2015);

b) en el borde nordpatagónico un evento compresivo en el entorno de los $261 \mathrm{Ma}$ (Guadalupiano tardío; Chernicoff et al., 2013) generaba el levantamiento del núcleo metamórfico del orógeno colisional de los Gondwánides Nordpatagónicos, y su denudación contribuía a la formación de gigantes lóbulos sedimentarios submarinos incorporados a la cuenca de Hespérides (Pángaro et al., 2016). Estos últimos autores señalan que la dirección de transporte de estos gigantes lóbulos sedimentarios era hacia el NNE y que, en conjunto, los mismos habrían cubierto una gran superficie que se extendía, en el sentido ESE, entre las cuencas de Carapacha y del Karoo oriental; asimismo, Pángaro et al. (2016, y referencias allí citadas) destacan que la deformación de estos sedimentos es post-triásica inferior (principalmente triásica inferior tardía);

c) asimismo, analizando el mismo lapso, o uno aún mayor (dentro del Paleozoico superior), resulta claro que las polaridades de la subducción parecen ser incongruentes entre sendas fajas orogénicas, con subducción hacia el este (margen continental sudamericano) en la Faja Sanrafaélica, y subducción hacia el sur (margen nordpatagónico) en los Gondwánides Norpatagónicos (también en su prolongación, en la faja de El Cabo; p. ej., Lindeque et al., 2011, fig. 12c);

d) este contraste entre las dos fajas gondwánicas es compatible con el diferente carácter de las mismas, i.e., acrecional en la Faja Sanrafaélica, y colisional en los Gondwánides Nordpatagónicos (terminología p. ej., de Cawood y Buchan, 2007);

e) Esta última diferencia, a su vez, permite dar una explicación satisfactoria a la distinta mineralización asociada a sendas fajas como, por ejemplo, la ausencia (Faja Sanrafaélica) o presencia (Gondwánides Norpatagónicos) de mineralización de wolframio en dichas fajas, indicando un criterio geotectónico para la exploración minera.

Por lo antedicho, el contraste evolutivo entre la Faja Sanrafaélica y la Faja Gondwánides Nordpatagónicos no parece poder ser explicado satisfactoriamente por rotación a escala subcontinental, como ha sido propuesto previamente (p. ej., Kleiman y Japas, 2009).

\section{CONCLUSIONES}

Se ha efectuado un aporte al esclarecimiento del contexto tectónico en que tuvo lugar el magmatismo pérmico-triásico de la provincia de La Pampa, Argentina. Así es que se definió un Corredor Magmático Pérmico-Triásico de La Pampa (CMPT-LP) (Figuras 1 y 7) ocurrido en un ambiente extensional intracratónico, por lo que sus productos se excluyen del Grupo Choiyoi, expuesto en la proximidad del CMPT. El ambiente anorogénico del CMPT-LP contrasta con el marco tectónico de la etapa tardía del Grupo Choiyoi propiamente dicho que si bien también corresponde a extensión tardío-pérmica a temprano-triásica, esta ocurre en el contexto orogénico propio del Arco Magmático Choiyoi.

Se dan a conocer nuevas edades U-Pb SHRIMP y la composición isotópica de Hf de dos unidades integrantes del CMPT-LP: (1) Sienogranito Chacharramendi, datado en $254.7 \pm 1.3 \mathrm{Ma}$ (Pérmico Superior, Lopingiano), con $\varepsilon_{\mathrm{Hf}}$ promedio de -5.68 y edad modelo de Hf de $1640 \mathrm{Ma}$; y (2) Riolita Lihue Calel datada en $239.3 \pm 1.5 \mathrm{Ma}$ (Triásico Medio), con y $\varepsilon_{\mathrm{Hf}}$ promedio de -5.56 y edad modelo de Hf de $1640 \mathrm{Ma}$. Estas determinaciones isotópicas, junto con datos isotópicos previos, indican que el CMPT-LP se formó a partir de un fundido que incorporó corteza statheriana tardía-calymmiana.

Los datos geoquímicos de roca total de las unidades datadas, analizados junto con datos geoquímicos previos, señalan el carácter anorogénico del corredor magmático CMPT-LP, destacándose su 


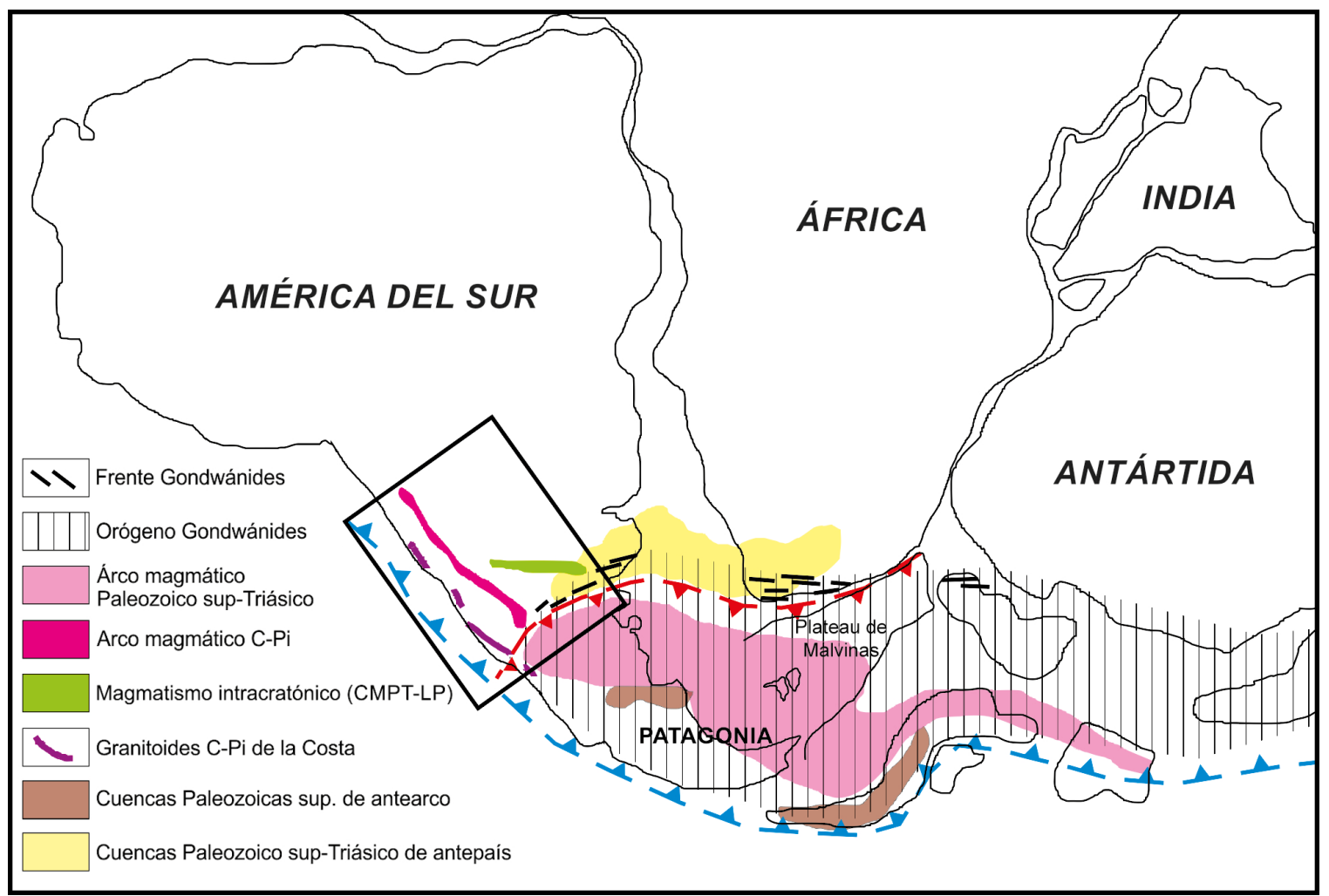

Figura 7. El Corredor Magmático Intracratónico Pérmico-Triásico de La Pampa (CMPT-LP) y elementos geológicos contemporáneos de la región (véase rectángulo insertado, correspondiente al área de la Figura 1), en el contexto paleogeográfico del continente de Gondwana. Base paleogeográfica tomada de Schmitt et al. (2017), Veevers (2004), Pángaro et al. (2016) y Werner (2006). C-Pi: Carbonífero-Pérmico Inferior.

consistencia con los granitos tipo $A_{1}$ de Eby (1992) debido a la similitud de sus fuentes con aquéllas a partir de las cuales se forman los basaltos de islas oceánicas (OIB), p. ej., Y/Nb $<1.2$, contrastante con los cocientes $\mathrm{Y} / \mathrm{Nb}>1.2$ hasta 5 de los granitoides de las dos fajas magmáticas Gondwánicas lindantes del CMPT-LP (fajas Sanrafaélica o Arco Choiyoi y Gondwánides Nordpatagónicos).

Asimismo, debido al carácter lindante y parcialmente contemporáneo que tienen las fajas magmáticas Gondwánicas Sanrafaélica (o San Rafael o Arco-retroarco Choiyoi) y Gondwánides Nordpatagónicos, respecto del Corredor Magmático Permo-Triásico de La Pampa presentado en este trabajo, se analizaron comparativamente sus marcos tectónicos de emplazamiento, concluyéndose que en vez de haber una continuidad física entre las mismas mediante una rotación a escala subcontinental, como se había propuesto anteriormente (p. ej., Kleiman y Japas, 2009), debe haber una discontinuidad o quiebre, que resulta particularmente conspicua en el lapso Guadalupiano a Lopingiano/ Triásico Inferior.

\section{AGRADECIMIENTOS}

Los circones fueron analizados en el SHRIMP II operado por el consorcio formado por la University of Western Australia (UWA) y el gobierno provincial de Western Australia, con apoyo del Australia Research Council. Las imágenes de electrones retrodispersados (backscattered electrons, BSE) fueron realizadas usando las instalaciones Centre for Microscopy, Characterization, and Analyses de la UWA. El Servicio Geológico-Minero Argentino (SEGEMAR) proveyó apoyo logístico para la realización del trabajo.

\section{REFERENCIAS}

Baldo, E., Casquet, C., Pankhurst, R.J., Galindo, C., Rapela, C.W., Fanning, C.M., Dahlquist, J.A., Murra, J., 2006, Neoproterozoic A-type granitic magmatism in the Western Sierras Pampeanas (Argentina): evidence for Rodinia break-up along a proto-Iapetus rift?: Terra Nova, 18(6), 388-394.

Baldo, E., Casquet, C., Colombo, F., Pankhurst, R.J., Galindo, G., Rapela, C.W., Dahlquist, J.A., Fanning, C.M., 2008, Magmatismo anorogénico Neoproterozoico (845 Ma) en las Sierras Pampeanas Occidentales de Maz y Espinal. ¿Nueva evidencia del rifting temprano de Rodinia?, en $17^{\circ}$ Congreso Geológico Argentino: San Salvador de Jujuy, Argentina, Vol. I, 181-182.

Barrionuevo, M., Arnosio, M., Llambías, E.J., 2013, Nuevos datos geocronológicos en subsuelo y afloramientos del Grupo Choiyoi en el oeste de La Pampa: implicancias estratigráficas: Revista de la Asociación Geológica Argentina, 70 (1), 31-39.

Blichert-Toft, J., Albarede, F., 1997, The Lu-Hf isotope geochemistry of chondrites and the evolution of the mantle-crust system: Earth Planetary Science Letters 148, 243-258.

Breitkreuz, C., Zeil, W., 1994, The Late Carboniferous to Triassic volcanic belt in northern Chile, en Reutter, K.J. (ed.), Tectonics of Southern Central Andes: Berlin, Springler-Verlag, 277-292.

Caminos, R., Llambías, E.J., Rapela, C.W., Parica, C.A., 1988, Late PaleozoicEarly Triassic magmatic activity of Argentina and the significance of new $\mathrm{Rb}-\mathrm{Sr}$ ages from northern Patagonia: Journal of South American Earth Sciences, 1(2), 137-145.

Casé, A.M., López-Escobar, L., Danieli, J.C., Schalamuk, A., 2008, Butalón igneous rocks, Neuquén, Argentina: Age, stratigraphic relationships and geochemical features: Journal of South American Earth Sciences, 26, 188-203.

Cawood, P.A., Buchan, C., 2007, Linking accretionary orogenesis with supercontinent assembly: Earth-Science Reviews 82, 217-256. 
Charrier, R., Ramos, V.A., Tapia, F., Sagripanti, L., 2014, Tectono-stratigraphic evolution of the Andean Orogen between 31 and $37^{\circ} \mathrm{S}$ (Chile and Western Argentina): Geological Society, London, Special Publications, doi 10.1144/ SP399.20.

Chernicoff, C.J., Zappettini, E., 2004, Geophysical evidence for terrane boundaries in south-central Argentina: Gondwana Research, 7(4), 1105-1116.

Chernicoff, C.J., Zappettini, E.O., Santos, J.O.S, Beyer, E. McNaughton, N.J., 2008, Foreland basin deposits associated with Cuyania terrane accretion in La Pampa province, Argentina: Gondwana Research, 13(2), 189-203.

Chernicoff, C.J., Zappettini, E.O., Santos, J.O.S., Belousova, E. y McNaughton, N.J., 2013, Combined U-Pb SHRIMP and Hf isotope study of the Late Paleozoic Yaminué Complex, Río Negro province, Argentina. Implications for the origin and evolution of the Patagonia composite terrane: Geoscience Frontiers, 4(6), 37-56.

Chernicoff, C.J., Zappettini, E.O., Santos, J.O.S., Belousova, E., McNaughton, N.J., 2016a, Combined U-Pb SHRIMP and Hf isotope study of Gondwanan igneous rocks at Chacharramendi and surroundings, La Pampa province, Argentina, en 10 South American Symposium on Isotope Geology: Puerto Vallarta, Jalisco, México, Actas, p. 132.

Chernicoff, C.J., Zappettini, E.O., Santos, J.O.S., McNaughton, N.J., 2016b, Las Matras pluton, Cuyania terrane, Argentina: is it truly Mesoproterozoic, or is it Early Permian derived from Mesoproterozoic?, en 35 International Geological Congress: Cape Town, Actas en GEOREF (American Geological Institute), Record ID: 0784662019.

de Elorriaga, E., 2010, Evaluación de los depocentros de la cuenca de Macachín, provincias de La Pampa y Buenos Aires, Argentina: Universidad Nacional de La Pampa, Argentina, Tesis Doctoral, 255 pp.

Domeier, M., Van del Voo, R., Tohver, E., Tomezzoli, R., Vizan, H., Torsvik, T., 2011, New Late Permian paleomagnetic data from Argentina: Refinement of the apparent polar wander path of Gondwana: GeochemistryGeophysics-Geosystems, https://doi.org/10.1029/2011GC003616.

Dristas, J.A., Hansteen, T., Deguillén, A.O., Kunis, S., 1998, Glass and $\mathrm{CO}_{2}$-dominated fluid inclusions in diopside-rich xenoliths and clinopyroxenesphenochrysts from comenditic trachyte, La Pampa province, Argentina, en 16 Geowissenschaftliches Lateinamerika Kolloquium: Bayreuth, Alemania, Terra Nostra, 98/5, 31.

Du Toit, A.L., 1927, A Geological Comparison of South America with South Africa. Carnegie Institution of Washington: Washington, EE.UU., Publication 381, $157 \mathrm{pp}$.

Eby, G.N., 1990, A review of their occurrence and chemical characteristics and speculations on their petrogenesis. Lithos 26, 115-134.

Eby, G.N., 1992, Chemical subdivision of the A-type granitoids: petrogenetic and tectonic implications: Geology, 20, 641-644.

Gregori, D.A., Grecco, L.E., Llambías, E.J., 2003, El intrusivo López Lecube: evidencias de magmatismo alcalino Gondwánico en el sector sudoeste de la provincia de Buenos Aires, Argentina: Revista de la Asociación Geológica Argentina, 58(2), 167-175.

Griffin, W.L., Pearson, N.J., Belousova, E.A., Jackson, S.R., van Achterbergh, E., O'Reilly, S.Y., Shee, S.R., 2000, The Hf isotope composition of cratonic mantle: LAM-MC-ICPMS analysis of zircon megacrysts in kimberlites: Geochimica et Cosmochimica Acta, 64, 133-147.

Griffin, W.L., Wang, X., Jackson, S.E., Pearson, N.J., O'Reilly, S.Y., Xu, X., Zhou, $X ., 2002$, Zircons chemistry and magma genesis in SE China: in situ analysis of Hf isotopes, Pingtan and Tonglu igneous complexes: Lithos, $61,237-269$.

Griffin, W.L., Belousova, E.A., Shee, S.R., Pearson, N.J., O’Reilly, S.Y., 2004, Archean crustal evolution in the northern Yilgarn Craton: U-Pb and Hf-isotope evidence from detrital zircons: Precambrian Research, 131, 231-282.

Griffin, W.L., Pearson, N.J., Belousova, E.A., Saeed, A., 2007, Reply to "Comment to short-communication 'Comment: Hf-isotope heterogeneity in zircon 91500 ' by W.L. Griffin, N.J. Pearson, E.A. Belousova and A. Saeed (Chemical Geology 233 (2006) 358-363)" by F. Corfu: Chemical Geology 244, 354-356.

Hawkesworth, C.J., Kemp, A.I.S., 2006, Evolution of the continental crust: Nature, 443, 811-817, https://doi.org/10.1038/nature05191.

Hervé, F., Fanning, C.M., Calderón, M., Mpodozis, C., 2014, Early Permian to Late Triassic batholiths of the Chilean Frontal Cordillera $\left(28^{\circ}-31^{\circ} \mathrm{S}\right)$ :
SHRIMP U-Pb zircon ages and Lu-Hf and O isotope systematic: Lithos, 184-187, 436-446.

Iizuka T., Hirata, T., 2005, Improvements of precision and accuracy in in situ $\mathrm{Hf}$ isotope microanalysis of zircon using the laser ablation-MC-ICPMS technique: Chemical Geology 220, 121-137.

Kay, S.M., Ramos, V.A., Mpodozis, C., Sruoga, P., 1989, Late Paleozoic to Jurassic silicic magmatism at the Gondwana margin: analogy to the Middle Proterozoic in North America?: Geology, 17, 324-328.

Keidel, J., 1916, La geología de las Sierras de la Provincia de Buenos Aires y sus relaciones con las montañas de Sudáfrica y Los Andes: Buenos Aires, Argentina, Ministerio de Agricultura de La Nación, Sección Geología, Mineralogía y Minería, Anales 11, 1-78.

Kleiman, L.E., Japas, M.S., 2009, The Choiyoi volcanic province at $34^{\circ}-36^{\circ}$ S (San Rafael, Mendoza, Argentina): implications for the late Paleozoic evolution of the southwestern margin of Gondwana: Tectonophysics, 473, 283-299.

Lagorio, S., Zappettini, E.O., Chernicoff, C.J., 2008, Stock Estancia El Trabajo: evidencias de magmatismo alcalino triásico en la provincia de La Pampa en 17 Congreso Geológico Argentino: San Salvador de Jujuy, Argentina, Actas, 1363-1364.

Limarino, C.O., Spalletti, L.A., 2006, Paleogeography of the upper Paleozoic basins of southern South America: an overview: Journal of South American Earth Sciences 22, 134-155.

Linares, E., Llambías, E.J., La Torre, C.A., 1980, Geología de la provincia de La Pampa, República Argentina y geocronología de sus rocas metamórficas y eruptivas: Revista de la Asociación Geológica Argentina, 35, 87-146.

Lindeque, A., de Wit, M.J., Ryberg, T., Weber, M., Chevallier, L., 2011, Deep crustal profile across the southern Karoo Basin and Beattie magnetic anomaly, South Africa: an integrated interpretation with tectonic implications: South African Journal of Geology, 114, 265-292.

Llambías, E.J., 1973, Las ignimbritas de la Sierra de Lihuel Calel, provincia de La Pampa, en 5 Congreso Geológico Argentino: Buenos Aires, Argentina, Actas, 4, 55-67.

Llambías, E.J., 1999, Las rocas ígneas gondwánicas, en Caminos, R. (ed.), Geología Argentina: Buenos Aires, Instituto de Geología y Recursos Minerales, 29(12), 349-376.

Llambías, E.J., Sato, A.M., 1990, El batolito de Colangüil (29-31º S) Cordillera Frontal de Argentina: estructura y marco tectónico: Revista Geológica de Chile 17, 89-108.

Llambías, E.J., Sato, A.M., 1995, El batolito de Colangüil: transición entre orogénesis y anorogénesis: Revista de la Asociación Geológica Argentina 50, 111-131.

Llambías, E.J., Kleiman, L.E., Salvarredi, J.A., 1993, El Magmatismo Gondwánico, en Ramos, V.A., (ed.), Geología y Recursos Naturales de Mendoza, XII Congreso Geológico Argentino y II Congreso de Exploración de Hidrocarburos: Mendoza, Argentina, Relatorio, 53-64.

Llambías, E.J., Quenardelle, S., Montenegro, T., 2003, The Choiyoi Group from central Argentina: a subalkaline transitional to alkaline association in the craton adjacent to the active margin of the Gondwana continent: Journal of South American Earth Sciences, 16, 243-257.

Llambías, E.J., Leanza, H.A., Carbone, O., 2007, Evolución tectonomagmática durante el Pérmico al Jurásico temprano en la Cordillera del Viento $\left(37^{\circ} 05^{\prime} \mathrm{S}-37^{\circ} 15^{\prime} \mathrm{S}\right)$ : nuevas evidencias geológicas y geoquímicas del inicio de la Cuenca Neuquina: Revista de la Asociación Geológica Argentina, 62(2), 217-235.

López-Gamundi, O.R., Fildani, A., Weislogel, A., Rossello, E., 2013, The age of the Tunas Formation in the Sauce Grande basin-Ventana foldbelt (Argentina): implications for the Permian evolution of the southwestern margin of Gondwana: Journal of South American Earth Sciences 45, 250-258.

Ludwig, K.R., 2001, Squid 1.02: A Users Manual: Berkeley Geochronology Centre, Special Publication. 2, 19 pp.

Ludwig, K.R., 2003, Isoplot 3.00, A Geochronological Took-kit for Excel: Berkeley Geochronology Center, Special Publication, 4, 67 pp.

Maniar, P.D., Piccoli, P.M., 1989, Tectonic Discrimination of Granitoids: Geological Society of America Bulletin, 101, 635-643.

Melchor, R.N. 2002, Depósitos continentales Eocenos en el sudoeste de La Pampa, Argentina: edad, estratigrafía y paleoambientes, en $15^{\circ}$ Congreso Geológico Argentino: El Calafate, Argentina, Actas, 1, 694- 698.

Mpodozis, C., Kay, S.M., 1992, Late Paleozoic to Triassic evolution of the 
Gondwana margin: Evidence from Chilean Frontal Cordilleran batholiths $\left(28^{\circ} \mathrm{S}\right.$ to $\left.31^{\circ} \mathrm{S}\right)$ : Geological Society of America Bulletin 104 (8), 999-1014.

Munizaga, F., Maksaev, V., Fanning, C.M., Giglio, S., Yaxley, G., Tassinari, C.C.G., 2008, Late Paleozoic-Early Triassic magmatism on the western margin of Gondwana: Collahuasi area, Northern Chile: Gondwana Research 13, 407-427.

Page, S., Zappettini, E. 1999, Magmatismo, provincias de Jujuy, Salta, Tucumán y Catamarca, en González-Bonorino, G., Omarini, R., Viramonte, J., (eds), 14 Congreso Geológico Argentino, Geología del Noroeste Argentino: Salta, Argentina, Relatorio, 241-253.

Pángaro, F., Ramos, V.A., Pazos, J., 2016, The Hesperides Basin: A Continentalscale upper Palaeozoic to Triassic basin in southern Gondwana: Basin Research, 28(5), 685-711.

Pankhurst, R.J., Rapela, C.W., Caminos, R., Llambías, E.J., Párica, C., 1992, A revised age for the granites of the central Somuncura batholith, North Patagonian Massif: Journal of South American Earth Sciences, 5, 321-325.

Pankhurst, R.J., Rapela, C.W., Fanning, C.M., Márquez, M., 2006, Gondwanide continental collision and the origin of Patagonia: Earth-Science Reviews, 76, 235-257.

Paton, D.A., Mortimer, E.J., Hodgson, N., Van Der Spuy, D., 2016, The missing piece of the South Atlantic jigsaw: when continental break-up ignores crustal heterogeneity, en Sabato Ceraldi, T., Hodgkinson, R.A., Backe, G. (eds). Petroleum Geoscience of the West Africa Margin: Geological Society, London, Special Publications, 438, 195-210.

Pearce, J.A., Harris, N.B.W., Tindle, A.G., 1984, Trace element discrimination diagrams for the tectonic interpretation of granitic rocks: Journal of Petrology 25, 956-983.

Poma, S., Zappettini, E.O., Quenardelle, S., Santos, J.O., Koukharsky, M., Belousova, E., McNaughton, N., 2014, Geochemistry, U-Pb SHRIMP zircon dating and $\mathrm{Hf}$ isotopes of the Gondwanan magmatism in NW Argentina: petrogenesis and geodynamic implications: Andean Geology, 41(2), 267-292.

Ramos, V.A., 2008, Patagonia: a Paleozoic continent adrift ?: Journal of South American Earth Sciences, 28, 235-251.

Rapela, C.W., Llambías, E.J., 1985, Evolución magmática y relaciones regionales de los complejos eruptivos de La Esperanza, provincia de Río Negro: Revista de la Asociación Geológica Argentina, 40, 4-25.

Rapela, C.W., Pankhurst, R.J., Llambías, E.J., Labudía, C., Artabe, A., 1996 "Gondwana" magmatism of Patagonia: inner cordilleran calk-alkaline batholiths and bimodal volcanic provinces, en Third ISAG: St Malo, Francia, 791-794.

Rocha-Campos, A.C., Basei, M.A., Nutman, A.P., Kleiman, L.E., Varela, R., Llambías, E., Canile, F.M., da Rosa, O., de C.R. da Rosa, 2011, 30 million years of Permian volcanism recorded in the Choiyoi igneous province (W Argentina) and their source for younger ash fall deposits in the Paraná Basin: SHRIMP U-Pb zircon geochronology evidence: Gondwana Research, 19, 509-523.

Rocher, S., Vallecillo, G., Castro de Machuca, B., Alasino, P., 2015, El Grupo Choiyoi (Pérmico temprano-medio) en la Cordillera Frontal de Calingasta, San Juan, Argentina: volcanismo de arco asociado a extensión: Revista Mexicana de Ciencias Geológicas, 32(3), 415-432.

Sato, A.M., Llambías, E.J., Shaw, S.E., Castro, C.E., 1990, El Batolito de Colangüil: modelo del magmatismo neopaleozoico de la provincia de San Juan, en Bordonaro, O. (ed.), 11 Congreso Geológico Argentino: San Juan, Argentina, Relatorio de Geología y Recursos Naturales de la provincia de San Juan, 100-122.

Sato, A.M., Tickyj, H., Llambías, E.J., Sato, K., 2000, The Las Matras tonalitictrondhjemitic pluton, central Argentina: Grenvillian-age constraints geochemical characteristics, and regional implications: Journal of South American Earth Sciences, 13, 587-610.

Sato, A.M., Llambías, E.J., Basei, M.A.S., Castro, C.A., 2015, Three stages in the Late Paleozoic to Triassic magmatism of southwestern Gondwana, and the relationships with the volcanogenic events in coeval basins: Journal of South American Earth Sciences, 63, 48-69.

Scherer, E., Münker, C., Mezger, K., 2001, Calibration of the Lutetium-Hafnium Clock: Science, 293(5530), 683-687.

Schmitt, R.S., Silva, E.A., Fragoso, R.A., Benedek, M.R., Richetti, P.C., Miller, W., Fernandes, G.L., Vasconcellos, I., Pereira, M.M., Costa, R.L., Collins, A.S., Reeves, C., 2017, The new geological map of Gondwana, scale 1:5000000 and its tectonic evolution - IFGCP-628 en 16th Puzzling out Gondwana International Conference, Bangkok, Tailandia, 12-21 noviembre, pre-1st edition, http://www.gondwana.geologia.ufrj.br/en/?page_id=26.

Spalletti, L.A., Limarino, C.O., 2017, The Choiyoi magmatism in south western Gondwana: implications for the end-permian mass extinction - a review: Andean Geology, 44(3), 328-338.

Sruoga, P., Llambías, E.J., 1992, Permo-Triassic leucorhyolitic ignimbrites at Sierra de Lihue Calel, La Pampa Province, Argentina: Journal of South American Earth Sciences, 5(2), 141-152.

Stern, R.A., 2001, New isotopic and trace-element standard for the ion microprobe: preliminary thermal ionization mass spectrometry (TIMS) $\mathrm{U}-\mathrm{Pb}$ and electron microprobe data; Radiogenic Age and Isotopic Studies: Report 14, Geological Survey of Canada. Current Research, F1, 11 pp.

Sun, S.S., 1980, Lead isotopic study of young volcanic rocks from mid-ocean ridges, ocean islands and island arcs: Philosophical Transactions of The Royal Society of London, Series A, Mathematical And Physical Sciences, 297, 409-445.

Thompson, R.N., 1982, Magmatism of the British Tertiary province: Scottish Journal of Geology, 18, 49-107.

Tickyj, H., Dimieri, L.V., Llambías, E. J., Sato, A.M., 1997, Cerro Los Viejos (382' $\left.\mathrm{S} / 64^{\circ} 26^{\prime} \mathrm{O}\right)$ : cizallamiento dúctil en el sudeste de La Pampa: Revista Asociación Geológica Argentina, 52, 311-321.

Tickyj, H., Tomezzoli, R., Chemale, F., Rapalini, A., 2010, Litología y edad de las volcanitas del Cerro El Centinela, provincia de La Pampa, en $10^{\circ}$ Congreso de Mineralogía y Metalogenia: Río Cuarto, Resúmenes.

Tomezzoli, R., Melchor, R., 2002, Evolución paleogeográfica del borde sudoccidental de Gondwana. Cuenca de Carapacha, La Pampa, Argentina, en 15 Congreso Geológico Argentino: El Calafate, Argentina, Asociación Geológica Argentina, CD-ROM, Artículo 329, 3 pp.

Tomezzoli, R.N., Tickyj, H., Woroszylo, M.E., 2011, Análisis de anisotropía de susceptibilidad magnética en el cerro El Centinela: Provincia de La Pampa, Argentina, Latinmag Letters, Proceedings 1, Special Issue D31, 1-5.

Uliana, M., Biddle, K., Cerdan, J. 1989, Mesozoic extension and the formation of Argentina sedimentary basins, en Tankard A.J., Balkwill, H. (eds.), Extensionals tectonics and stratigraphy of the North Atlantic margins: American Association of Petroleum Geologists, Memoir 46, 599-614.

Varela, R., Gregori, D.A. González, P.D., Basei, M.A.S., 2015, Caracterización geoquímica del magmatismo de arco devónico y carbonífero-pérmico en el noroeste de Patagonia, Argentina: Revista de la Asociación Geológica Argentina, 72(3), 419-432.

Webster, R.E., Chebli, G.A., Fritz Fischer, J., 2004, General Levalle basin, Argentina: A frontier Lower Cretaceous rift basin: AAPG Bulletin, 88(5), 627-652.

Werner, M., 2006, The stratigraphy, sedimentology and age of the Late Paleozoic Mesosaurus Inland Sea, SW Gondwana - New Implications from studies on sediments and altered pyroclastic layers of the Dwyka and Ecca Group (Lower Karoo Super group) in southern Namibia: Würzburg, University of Würzburg, Ph.D. tesis, 425 pp.

Whalen, J.B., Curie, K.L., Chappel, B.W., 1987, A-type granites: Geochemical characteristics, discrimination and petrogenesis: Contributions to Mineralogy and Petrology, 95(4), 407-419.

Wiedenbeck, M., Alle, P., Corfu, F., Griffin, W.L., Meier, F., Oberli, F., Von Quadt, A., Roddick, J.C., Spiegel, W., 1995, Three natural zircon standards for U-Th-Pb, Lu-Hf, trace element, and REE analyses: Geostandards Newsletter, 19, 1-23.

Zappettini, E.O., Blasco, G. 1998, Hoja Geológica 2569-II Socompa, provincia de Salta: Buenos Aires, Servicio Geológico Minero Argentino (SEGEMAR), Boletín 160, 83 pp.

Zappettini, E.O., Crosta, S., Rubinstein, N., Segal, S., 2015, Revisión de modelos de mineralización vinculados a fallas en ambiente de rifting. Implicancias metalogenéticas relacionadas al rifting mesozoico en Argentina: Buenos Aires, Argentina, Servicio Geológico-Minero Argentino, Serie Contribuciones Técnicas, Recursos Minerales, 37, 1-63.

Manuscrito recibido: junio 5, 2018

Manuscrito corregido recibido: agosto 17, 2018

Manuscrito aceptado: septiembre 7, 2018 